\title{
Medical Image Segmentation Using Independent Component Analysis-Based Kernelized Fuzzy $c$-Means Clustering
}

\author{
Yao-Tien Chen \\ Department of Applied Mobile Technology, Yuanpei University of Medical Technology, Hsinchu 30015, Taiwan \\ Correspondence should be addressed to Yao-Tien Chen; ytchen@mail.ypu.edu.tw
}

Received 26 July 2016; Revised 24 October 2016; Accepted 24 November 2016; Published 4 January 2017

Academic Editor: Masoud Hajarian

Copyright ( 2017 Yao-Tien Chen. This is an open access article distributed under the Creative Commons Attribution License, which permits unrestricted use, distribution, and reproduction in any medium, provided the original work is properly cited.

\begin{abstract}
Segmentation of brain tissues is an important but inherently challenging task in that different brain tissues have similar grayscale values and the intensity of a brain tissue may be confused with that of another one. The paper accordingly develops an ICKFCM method based on kernelized fuzzy $c$-means clustering with ICA analysis for extracting regions of interest in MRI brain images. The proposed method first removes the skull region using a skull stripping algorithm. Through ICA, three independent components are then extracted from multimodal medical images containing T1-weighted, T2-weighted, and PD-weighted MRI images. As $M R I$ signals can be regarded as a combination of the signals from brain matters, ICA can be used for contrast enhancement of MRI images. Finally, the three independent components are utilized as inputs by KFCM algorithm to extract different brain tissues. Relying on the decomposition of a multivariate signal into independent non-Gaussian components and using a more appropriate kernel-induced distance for fuzzy clustering, the proposed method is capable of achieving greater reliability in both theory and practice than other segmentation approaches. According to the experiment results, the proposed method is capable of accurately extracting the complicated shapes of brain tissues and still remaining robust against various types of noises.
\end{abstract}

\section{Introduction}

Along with the rapid advancement of medical imaging technologies, there has been a constant rise in the demand for more sophisticated visual means for inspection of anatomic structures, identification of disease, examination of the characteristics in a region of interest, and registration and reconstruction of images. Of the technologies developed to meet the demand, image segmentation plays an indispensable role. Medical images are mostly limited by low contrast, high noise, intensity inhomogeneity, and other imaging ambiguities greatly complicating the task of segmentation. In addition, compared with other types of images, medical images tend to pose greater difficulty in shape recovery. More accurate and effective techniques are therefore needed to assist medical image segmentation. Image segmentation refers to the technique that partitions an image into different regions and is typically used to identify regions of interest. Several algorithms have been reported in the literature for image segmentation, such as independent component analysis $[1,2]$, fuzzy clustering [3], level set method [4, 5], Markov random field [6], and neural network [7], each of which has its advantages and disadvantages.

Most medical images are acquired from special acquisition modalities, including magnetic resonance imaging (MRI), computed tomography $(C T)$, single photon emission tomography (SPECT), positron emission tomography (PET), and ultrasounds (US). Because of its advantages over other diagnostic imaging techniques [8], MRI has been extensively used for image segmentation. To reduce the uncertainty widely present in medical images, this study strives to integrate independent component analysis (ICA), kernelized fuzzy $c$-means clustering (KFCM), and image preprocessing as a computational technique for use in medical image segmentation.

A statistical and computational technique for revealing hidden factors that underlie sets of random variables, measurements, or signals, ICA defines a generative model for the observed multivariate data, which is assumed to be mixtures of some unknown latent variables. It was originally developed as a means to solve the problem of blind source separation (BSS) [9]. BSS refers to the problem of finding the original 
source signals from available mixtures, without having prior knowledge of the mixing mechanisms. For performing unsupervised classification, the data are modelled as a mixture of classes described by linear combinations of independent nonGaussian densities. ICA has been successfully used to separate multivariate signals, yet two statistical limitations should be met. Lee and his colleagues [10] proposed the ICA mixture model (ICAMM) to overcome one of the ICA limitations, which is the assumption that the signal sources are independent. In such approach, the assumption is relaxed by using the concept of mixture models. The ICAMM algorithm finds the independent components and the mixing matrix for each class and computes the class membership probability for each pattern in the dataset. The learning rules for the ICAMM were derived using gradient ascent method to maximize the loglikelihood data function. Attempting to improve the ICAMM performance, Oliveira and Romero [1] proposed an enhanced ICA mixture model (EICAMM), which implements some modifications on the original ICAMM algorithm learning rules. EICAMM algorithm is similar to the original ICAMM algorithm as both are based on an information-maximization approach proposed by Bell and Sejnowski [11].

$I C A$ and its extension have been extensively applied in many research fields [12]. Jenssen and Eltoft [2] used ICA image model to generate a data dependent filter bank for texture segmentation. The filter bank consists of the ICA basis images which are learned from textured images. With the basis images capable of capturing the inherent structure of the texture, their method is able to segment the texture image. Tateyama et al. [13] proposed a method based on kernel independent component analysis (KICA) for classification of $M R I$ datasets. KICA, a nonlinear approach for extracting independent components of images, appeared to produce significant enhancement in brain $M R I$ datasets, and their experiment found the KICA-based classification method able to effectively classify brain tissues.

Among the other methods developed for MRI image segmentation, fuzzy clustering is of considerable benefits thanks to its ability to retain much more information from the original image than hard segmentation methods. Clustering algorithms attempt to classify a pixel to a tissue class by applying the notion of similarity to the class; they can thus be adopted for image segmentation. Unlike the crisp $K$-means clustering algorithm [14], the fuzzy $c$-means (FCM) algorithm allows pixels to belong to multiple clusters with varying degrees of membership; algorithms based on fuzzy clustering are therefore particularly suitable for handling an uncertainty problem concerning segmentation of multimodal medical images. Masulli and Schenone [15], after conducting a review of different clustering algorithms containing FCM algorithm, maximum entropy principle-based fuzzy clustering ( $M E P$ FC) [16], and two versions of possibilistic $c$-means algorithms (PCMs) [17, 18], introduced their clustering algorithm named possibilistic neurofuzzy $c$-means algorithm (PNFCM). The KFCM algorithm $[19,20]$ was realized by replacing the original Euclidean distance in the FCM algorithm with a kernelinduced distance and a spatial penalty on the membership functions. Kannan et al. [21] proposed a kernel-induced fuzzy $c$-means based on hypertangent function for segmenting breast MRI. The algorithm was developed by integrating kernel, hypertangent function, and Lagrangian methods with the basic objective function of the FCM algorithm to obtain proper effective segmentation. Gupta et al. [3] presented a hybrid segmentation method utilizing both features of Gaussian kernel-induced fuzzy c-means (GKFCM) clustering [22] and active contour model driven by region scalable fitting $(R S F)$ energy function [23] for ultrasound medical images. In this method, the result obtained from the GKFCM method is used to initialize the contour that spreads to identify the estimated regions. GKFCM also helps to estimate the controlling parameters used in the curve evolution process and achieve better results with the kernel-induced distance replaced by the Euclidean distance. The RSF formulation is responsible for attracting the contour toward the object boundaries and it does not require the reinitialization process when the level set curve is evolving. Gong et al. [24] proposed an improved FCM, called KWFLICM, by introducing a tradeoff weighted fuzzy factor and a kernel metric. The tradeoff weighted fuzzy factor depending on the space distance of all neighboring pixels and their gray-level difference is introduced into its objective function to guarantee noise insensitiveness and image detail preservation. To enhance segmentation robustness to noise and outliers, KWFLICM algorithm further incorporates a kernel distance measure in its objective function. The KWFLICM algorithm, which can accurately estimate the damping extent of neighboring pixels, yields good results in many cases of image segmentation and maintains fine robustness to various types of noises.

Despite their advantages in yielding regions more homogeneous than other crisp methods, reducing spurious blobs, and removing noisy spots, most of the fuzzy methods mentioned above still require prior knowledge about the number of clusters in the data that may not be known for new data. Instead of improving segmentation accuracy via enhancing the kernel function, eighteen different validity criteria [25] have been implemented to estimate the number of clusters to help achieve high-accuracy segmentation of a given image when they are performed with KFCM or SKFCM algorithm $[26,27]$.

\section{Method}

As shown in Figure 1, the proposed segmentation method comprises three stages: skull stripping, independent component analysis, and kernelized fuzzy $c$-means clustering.

2.1. Skull Stripping. An essential step in most quantitative analyses of brain structure is removing the skull region, that is, the bones of skull and meninges, from the original image. To complete the task, a threshold that can separate brain and nonbrain tissues is calculated according to the histogram computed by image grayscale. With the thresholding, a binary image can be created. After creating the binary image, we eliminate isolated fake objects and holes by morphological operations from the binary image to produce another binary image, called binary morphological image (i.e., we remove the isolated fake objects outside the white portion and fill the 


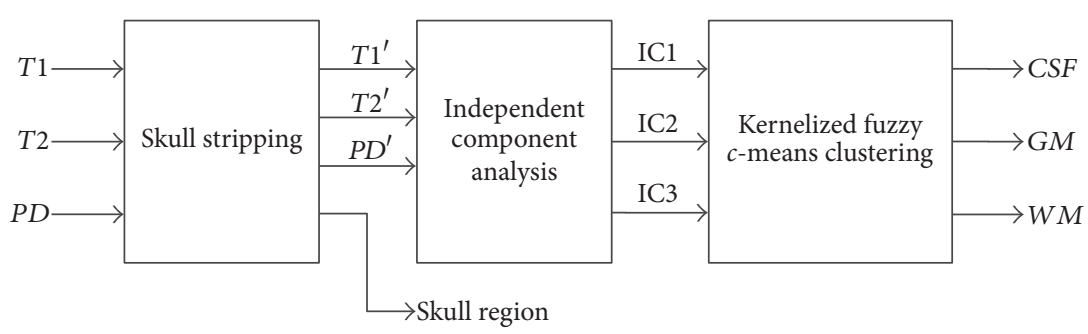

FIGURE 1: The proposed segmentation method integrating skull stripping, ICA, and KFCM algorithm.

holes inside the white portion in the first binary image). To remove the region boundaries from foreground pixels (i.e., white pixels), erosion operation is performed in the binary morphological image to obtain a binary eroded image. The last step is to mask the grayscale image using the binary eroded image. The mask operation leads to the creation of the skull stripped image; the skull image can then be obtained. The skull stripping algorithm is summarized as follows.

Step 1. Calculate a threshold that can separate brain and nonbrain tissues based on the histogram computed by image grayscale.

Step 2. Apply the threshold to create a binary image.

Step 3. Eliminate isolated fake objects and holes from the binary image to create a binary morphological image.

Step 4. Remove $p$ layers of pixels to obtain a binary eroded image, where parameter $p$ is a constant and $10 \leq p \leq 12$.

Step 5. Mask the grayscale image using the binary eroded image to achieve the skull stripped image.

2.2. Independent Component Analysis. In this section, we first briefly review the principle of the independent component analysis and then apply the ICA algorithm to analyze the multimodal $M R I$ images, for extracting three independent components.

When the ICA algorithm is applied to the segmentation of brain tissues, three types of images, T1-weighted, T2weighted, and $P D$-weighted images, are regarded as the observed signals and represented by $x_{n}, n=1,2,3$, respectively. The set of these images is described as

$$
X=\left[x_{1}, x_{2}, x_{3}\right]^{T} .
$$

Brain tissues containing WM, GM, and CSF can be regarded as the source signals, respectively, represented by $s_{p}, p=1,2,3$. The set of these brain tissues is described as

$$
S=\left[s_{1}, s_{2}, s_{3}\right]^{T}
$$

The relation between images $X$ and brain tissues $S$ can then be represented as

$$
X=A S \text {, }
$$

where $A$ is an unknown mixing matrix of size $3 \times 3$. If we can obtain the matrix $A$, then the brain tissues can be obtained by the calculation of inverse matrix $A$ as

$$
S=A^{-1} X
$$

However, according to the known condition, matrix $A$ is still unknown. By measurement, analysis of statistical independence of observed signals $X$, and through mathematical operation, an approximation matrix $A$ can be estimated. With the inverse matrix $W=A^{-1}$ (called demixing matrix) calculated, the approximation of $S$, denoted by $\widehat{S}$, can be predicted, and $\widehat{S}$ stands for the components of brain tissues. The approximation of $S$ (i.e., the components of brain tissues to be segmented) is presented as

$$
S \approx \widehat{S}=W X .
$$

In the paper, the FastICA algorithm is adopted to calculate the matrix $W$ and to further analyze each pixel in the multispectral image for correct classification of brain tissue.

FastICA $[12,28]$ uses a gradient descent method to maximize a measure of non-Gaussianity, thus accelerating the estimation of the independent components of source signals. Before applying this algorithm, two preprocessing steps, centering and whitening, are usually necessary.

(i) Center the Data. The first step is to center the observed data, that is, to subtract the mean $E\{X\}$ from the observed data $X=A S$, where $A$ is a mixing matrix and $S$ is a latent source signal (data), so it has zero mean.

(ii) Whiten the Data Using Principal Components Analysis (PCA). After centering, PCA is performed on the zero-mean data to constrain the search of weights to an orthogonal space, that is, to transform observed data $X$ so that they are uncorrelated and have unit variance. Whitening is necessary as it makes it possible to search the independent components one by one and prevents the estimated weight vectors from converging to the same optimum. Moreover, finding the weight vectors one by one greatly improves the convergence speed.

The FastICA algorithm uses a gradient descent method to optimize the negentropy equation:

$$
J(y)=[E(G(y))-E(G(v))]^{2},
$$

where $y$ is a normalized random variable with zero mean and unit variance; $E$ is a mean function; $G$ is any nonquadratic 
function; and $v$ is a standard Gaussian variable with zero mean and unity variance. In the paper, the algorithm for FastICA is described as follows.

Step 1. Choose an initial random weight vector $w$.

Step 2. Update demixing matrix $W$ by $w_{n+1}=E\left(X g\left(w_{n}^{T} X\right)\right)-$ $E\left(g^{\prime}\left(w_{n}^{T} X\right)\right) w_{n}$, where $g(x)=\tanh (x)$.

Step 3. Normalize weight matrix $w$ by $w_{n+1}=w_{n+1} /\left\|w_{n+1}\right\|$.

Step 4. Set $n \leftarrow n+1$, and go back to Step 2 if $\left\|w_{n+1}-w_{n}\right\| \geq \varepsilon$, where $\varepsilon \geq 0$ is the smallest constant.

After applying the FastICA algorithm to analyze the multimodal $M R I$ images (a linear combination of T1-weighted, T2-weighted, and $P D$-weighted $M R I$ images), we can extract three independent components. Compared with the original multimodal images, signals in two of the three independent components have been contrast enhanced. Therefore, the first independent component is used for the segmentation of WM and $G M$, while the second one is used for the segmentation of CSF to obtain more accurate segmentation results.

2.3. Kernelized Fuzzy c-Means Clustering. A kernelized FCM $(K F C M)$ algorithm $[20,25]$ is constructed with the following objective function as

$$
J_{m}=\sum_{i=1}^{n} \sum_{j=1}^{c} u_{i j}^{m}\left\|\Phi\left(x_{i}\right)-\Phi\left(c_{j}\right)\right\|^{2}, \quad 1 \leq m \leq \infty,
$$

where fuzzifier $m$ is a real number greater than $1, \Phi$ is an implicit nonlinear mapping, and $x_{i}$ and $c_{j}$ are, respectively, the $i$ th measured data and center of the $j$ th cluster in the original space. The implicit nonlinear mapping is defined as follows. From the data space to the mapped $d$ feature space, $\Phi: X \rightarrow F\left(x \in R^{p} \rightarrow \Phi(x) \in R^{d}, d>p\right)$, a dataset $\left\{x_{1}, x_{2}, \ldots, x_{n}\right\} \subseteq X$ (input data has low $d$-dimensional vector space) is mapped into a potentially much higher $p$ dimensional feature space or inner product $F$. The purpose of this mapping is to turn the original nonlinear problem in the input space into a potentially linear one in a rather high dimensional feature space. A kernel $K(x, y)$ in the feature space can be represented as

$$
K(x, y)=\langle\Phi(x), \Phi(y)\rangle,
$$

where $\langle\Phi(x), \Phi(y)\rangle=\Phi^{T}(x) \Phi(y)$ denotes the inner product operation. In the study, Gaussian radial basis function (GRBF) kernel [29] is adopted and described as

$$
K(x, y)=\exp \left(-\frac{\|x-y\|^{2}}{\sigma^{2}}\right),
$$

where $\sigma$ is a parameter of Gaussian kernel.

Based on the kernel substitution trick, we have

$$
\begin{gathered}
\left\|\Phi\left(x_{i}\right)-\Phi\left(c_{j}\right)\right\|^{2}= \\
K\left(x_{i}, x_{i}\right)-2 K\left(x_{i}, c_{j}\right) \\
+K\left(c_{j}, c_{j}\right) .
\end{gathered}
$$

Since $K(x, c)=\exp \left(-\|x-c\|^{2} / \sigma^{2}\right), K\left(x_{i}, x_{i}\right)=1, K\left(c_{j}, c_{j}\right)=$ 1 , and $\Phi^{T}\left(x_{i}\right) \Phi\left(c_{j}\right)=\Phi\left(x_{i}\right) \Phi^{T}\left(c_{j}\right)$, (7) can be rewritten as

$$
J_{m}=2 \sum_{i=1}^{n} \sum_{j=1}^{c} u_{i j}^{m}\left(1-K\left(x_{i}, c_{j}\right)\right), \quad 1 \leq m \leq \infty .
$$

As shown in a previous study [30], the GRBF kernel tends to achieve better segmentation results on simulated $M R I$ images corrupted by noise and other artifacts than polynomial kernel. Thus, the GRBF kernel is adopted as a distance metric replacing the standard Euclidean distance for reliable KFCM clustering.

Like the FCM algorithm, the objective function $J_{m}$ in (11) can be minimized under the constraint of $U$ (i.e., $\sum_{j=1}^{c} u_{i j}=$ 1). The fuzzy membership matrix $U$ can thus be obtained by

$$
u_{i j}=\frac{\sum_{k=1}^{c}\left(1-K\left(x_{i}, c_{k}\right)\right)^{1 /(m-1)}}{\left(1-K\left(x_{i}, c_{j}\right)\right)^{1 /(m-1)}}
$$

where $c$ is the number of clusters; $c_{j}$ and $c_{k}$ are the cluster centers of fuzzy groups $j$ and $k$, respectively; and the parameter $m$ (fuzzifier) is a weighting exponent on each fuzzy membership. The cluster center $c_{j}$ can be obtained by

$$
c_{j}=\sum_{i=1}^{n} \frac{u_{i j}^{m} K\left(x_{i}, c_{j}\right) x_{i}}{u_{i j}^{m} K\left(x_{i}, c_{j}\right)} .
$$

\section{Results and Discussion}

All the algorithms were implemented in MATLAB and C\# programming language. Simulated $M R I$ images downloaded from BrainWeb website [31] were used as the test images. To obtain more realistic experiment results, medical images provided by the National Library of Medicine [32] and the Department of Medical Imaging and Radiological Technology at YPU (the Yuanpei University of Medical Technology) [33] had also been used.

Five parts of the experiments are reported: preprocessing for skull stripping, extraction of independent components by ICA, qualitative comparison with state-of-the-art segmentation methods, quantitative comparison of segmentation accuracy, and image segmentation using real medical images.

Figure 2 presents three types of original simulated T1weighted, T2-weighted, and $P D$-weighted $M R I$ brain images, and the image size is $181 \times 217$ pixels. At first, the skull stripping algorithm was used to strip the skull region of the brain image. Original T1-weighted $M R I$ brain image, binary image, binary morphological image, binary eroded image, skull region image, and skull stripped image are shown in Figures 3(a)-3(f), respectively. As indicated, the approach can accurately strip the skull region from the examined brain image. Moreover, when applied to other types of medical images, the skull stripping was also capable of achieving fine results. 


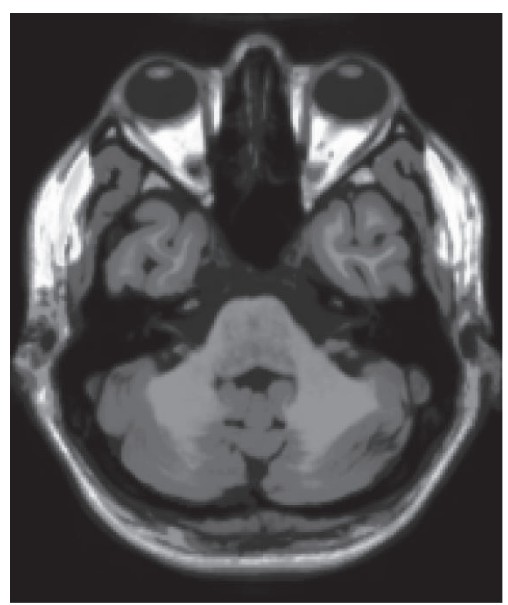

(a)

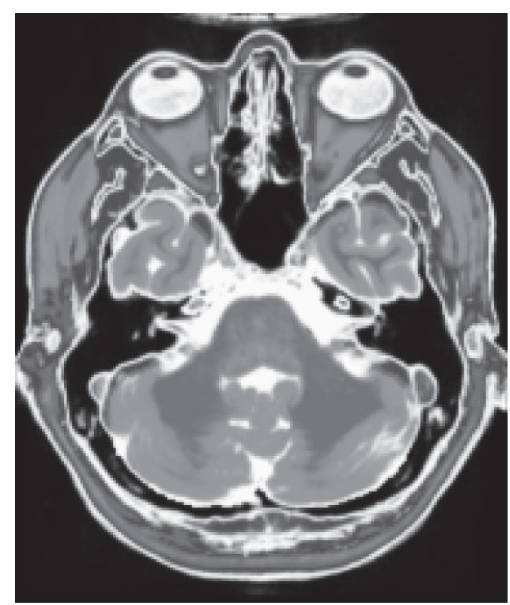

(b)

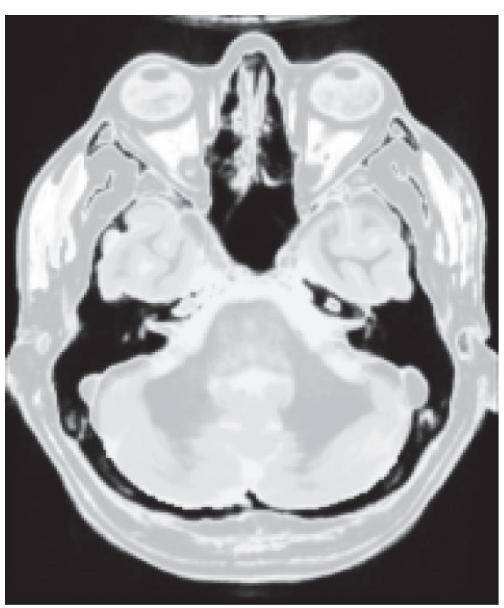

(c)

FIgURE 2: T1-weighted, T2-weighted, and PD-weighted MRI brain images.

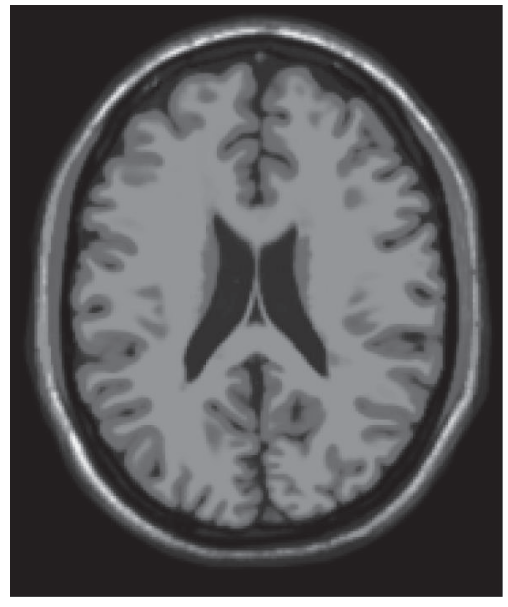

(a)

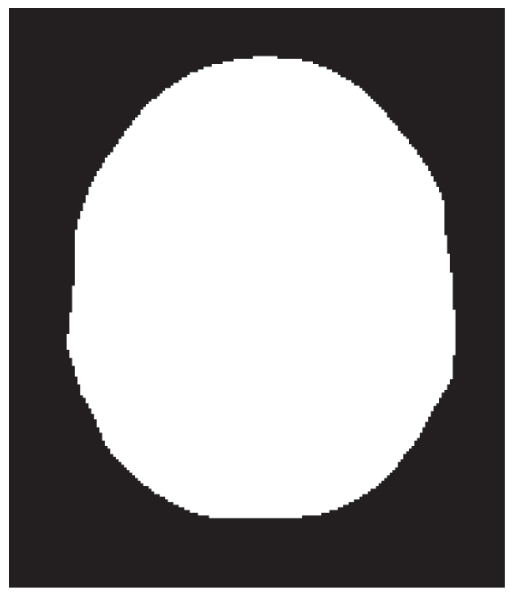

(d)

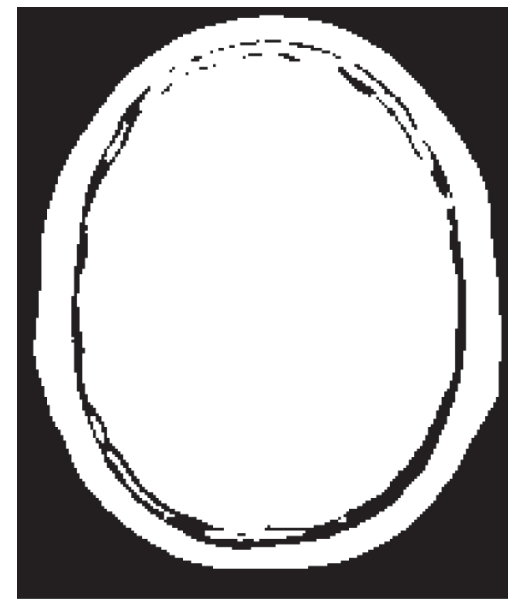

(b)

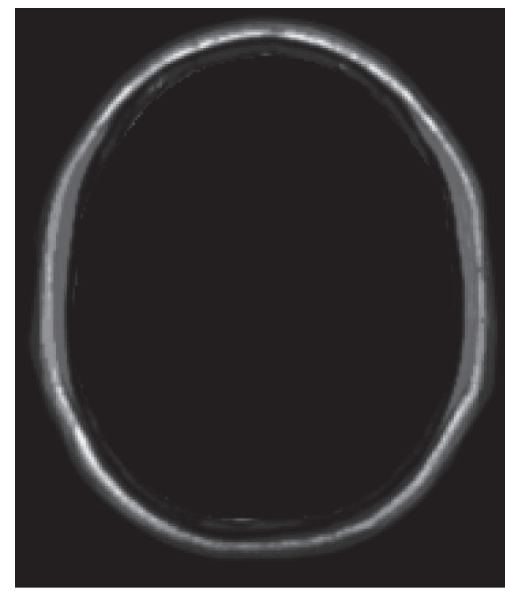

(e)

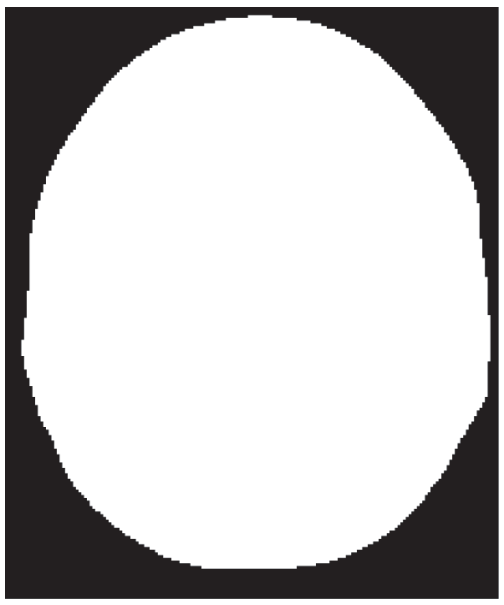

(c)

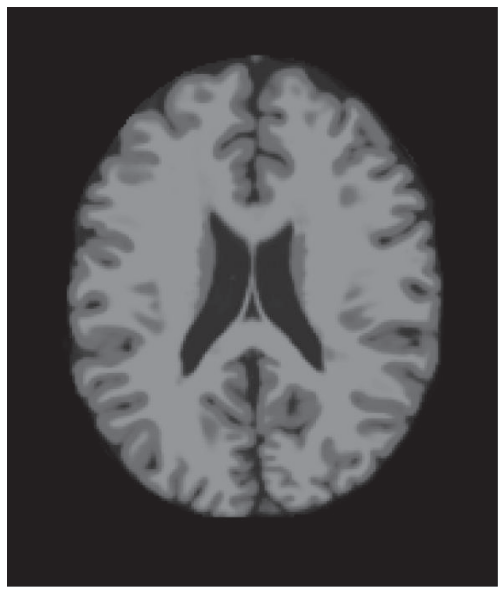

(f)

FIGURE 3: The skull stripping. (a) Original T1-weighted MRI brain image. (b) Binary image. (c) Binary morphological image. (d) Binary eroded image. (e) Skull region image. (f) Skull stripped image. 
To demonstrate that ICA can be adopted for contrast enhancement of MRI images, the FastICA algorithm was used to extract the independent components in multimodal medical images (a linear combination of T1-weighted, T2weighted, and $P D$-weighted $M R I$ images). Figures 4(a)-4(c) present three simulated images, T1-weighted, T2-weighted, and $P D$-weighted $M R I$ brain images of the 94th slice, where image size $=181 \times 217$, slice thickness $=1 \mathrm{~mm}$, and noise $=$ $0 \%$ (the noise was calculated relative to the brightest tissue), with intensity nonuniformity ("RF") with $0 \%$. Figures $4(\mathrm{~d})-$ 4(f) show the results of the images without skull region after centering and whitening. Visual inspection finds only a slight difference between these two sets of images (two rows in Figure 4). Using the FastICA algorithm to extract the independent components and then rescaling the intensity range of the independent components, we obtained three independent components as shown in Figures 4(g)-4(i). It can be seen that the contrast of $W M$ is enhanced in the first component (Figure $4(\mathrm{~g})$ ), while the contrast of CSF is enhanced in the second component (Figure 4(h)). Similarly, as in the previous experiment, the three simulated images were added by different noises with 3\% and 5\% as shown in Figures 5 and 6, respectively; the FastICA algorithm was then used to extract the independent components. As indicated by the results of applying the FastICA algorithm, ICA can enhance the contrast of images despite the presence of noise corruption. Moreover, after the algorithm was applied, a small part of the noise in the independent components disappeared as shown in Figures $5(\mathrm{~g}), 5(\mathrm{~h}), 6(\mathrm{~g})$, and $6(\mathrm{~h})$.

Before the experiments of qualitative and quantitative comparisons of different methods, we listed the ground truth of brain tissue segmentation of the simulated $M R I$ images of the 94th slice obtained from the same BrainWeb website [31] in Figure 7. It presents the several different anatomical regions in the human brain in terms of WM, GM, CSF, muscle/skin, skin, skull, connective tissue, fat, glial matter (glial matter is a connective tissue in the brain and a CSF-WM interface with an image intensity intermediate between $W M$ and $G M$ ), and background. To qualitatively compare segmentation performance, a total of six methods were used for the simulated $M R I$ images: $k$-means, FCM, KFCM, KWFLICM, FCM with ICA analysis (ICFCM), and the proposed KFCM with ICA analysis (ICKFCM).

In our experiments, a brain image was segmented into only four major regions: WM, GM, CSF plus glial matter, and the skull region. Figure 8 illustrates the case of tissue segmentation when the simulated MRI images do not suffer noise corruption. The segmentation results are reported in Figures 8(a)-8(c) using $k$-means algorithm, Figures $8(d)-8(f)$ using FCM algorithm, Figures $8(\mathrm{~g})-8(\mathrm{i})$ using KFCM algorithm, Figures 8(m)-8(o) using KWFLICM algorithm, Figures $8(\mathrm{p})-$ 8(r) using ICFCM algorithm, and Figures $8(\mathrm{~s})-8(\mathrm{u})$ using ICKFCM algorithm. The three independent components extracted from multimodal medical images are also shown in Figures $8(j)-8(1)$ for visual inspection.

Figure 9 illustrates the case of tissue segmentation with the simulated MRI images at a noise level of 3\%. The segmentation results are reported in Figures 9(a)-9(c) using $k$ means algorithm, Figures 9(d)-9(f) using FCM algorithm,
Figures 9(g)-9(i) using KFCM algorithm, Figures 9(m)-9(o) using KWFLICM algorithm, Figures 9(p)-9(r) using ICFCM algorithm, and Figures 9(s)-9(u) using ICKFCM algorithm. The three independent components extracted from multimodal medical images are also shown in Figures 9(j)-9(1) for visual inspection. Figure 10 presents another case of tissue segmentation when the simulated $M R I$ images have a 5\% noise level. The segmentation results are reported in Figures 10(a)-10(c) using $k$-means algorithm, Figures 10(d)10(f) using FCM algorithm, Figures 10(g)-10(i) using KFCM algorithm, Figures 10(m)-10(o) using KWFLICM algorithm, Figures $10(\mathrm{p})-10(\mathrm{r})$ using ICFCM algorithm, and Figures $10(\mathrm{~s})-10(\mathrm{u})$ using ICKFCM algorithm. The three independent components extracted from multimodal medical images are also shown in Figures $10(\mathrm{j})-10(\mathrm{l})$ for visual inspection. In all the equations of segmentation methods based on fuzzy clustering, $m=2, \varepsilon=0.00001$, and maximum iteration $=$ 80. In addition, Gaussian kernel parameter $\sigma=150$, and $c$ (i.e., the number of clusters) is normally not known in advance. However, as a brain image without skull region is usually delineated into three regions, $c$ was set at 3 . As indicated by the results of the qualitative comparison, no matter in the noiseless or noise case, the proposed method (i.e., ICKFCM algorithm) emerged to be capable of extracting the brain tissues in a more accurate manner than the other four comparable methods.

For further visual comparison of segmentation results (Figure 8) based on ICKFCM, KWFLICM, and KFCM algorithms, Figure 11 presents an example illustrating the ability of the proposed method for brain tissue extraction, where red boxes denote the regions of interest (ROI). Figure 11(a) shows the ground truth of GM. Figures 11(b)-11(d) show the segmentation results of GM using ICKFCM, KWFLICM, and KFCM algorithms. Figures 11(e) and 11(i) show the enlargement regions of ROI in ground truth of GM. Figures 11(f) and 11(j) show the enlargement regions of ROI in Figure 11(b). Figures $11(\mathrm{~g})$ and $11(\mathrm{k})$ show the enlargement regions of $R O I$ in Figure 11(c). Figures 11(h) and 11(l) show the enlargement regions of ROI in Figure 11(d). As indicated by the extraction results, the proposed ICKFCM method can accurately extract the complicated shapes of $G M$ with the longitudinal fissure clearly delineated. Moreover, when applied to extract other brain tissues such as WM, CSF, and glial matter, the proposed method can also achieve fine segmentation results compared with other segmentation methods.

To quantitatively evaluate the accuracy of the segmentation methods, Jaccard coefficient (measure similarity) [34] was used as a measure of similarity between the region delineated by the computerized method and the ground truth, and it is defined as

$$
J\left(S_{g}, S_{C}\right)=\frac{\left|S_{g} \cap S_{C}\right|}{\left|S_{g} \cup S_{C}\right|},
$$

where $S_{g}$ refers to the binary mask of the ground truth, $S_{C}$ indicates the region segmented by the computerized method, and $|\cdot|$ denotes the cardinality of a set. The value of this measure varies between 0 and 1 , and a higher value of Jaccard 


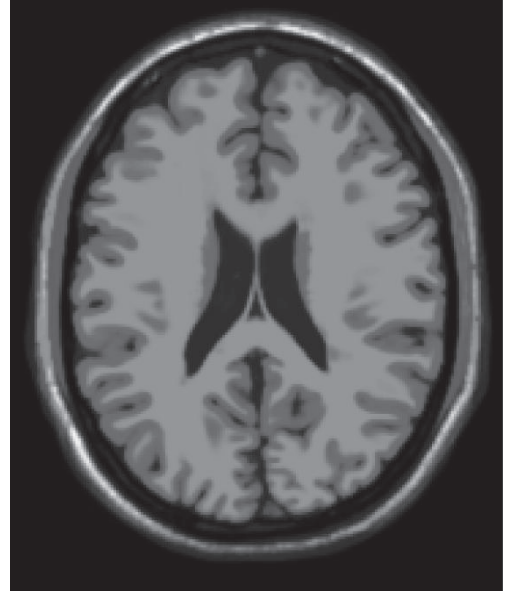

(a)

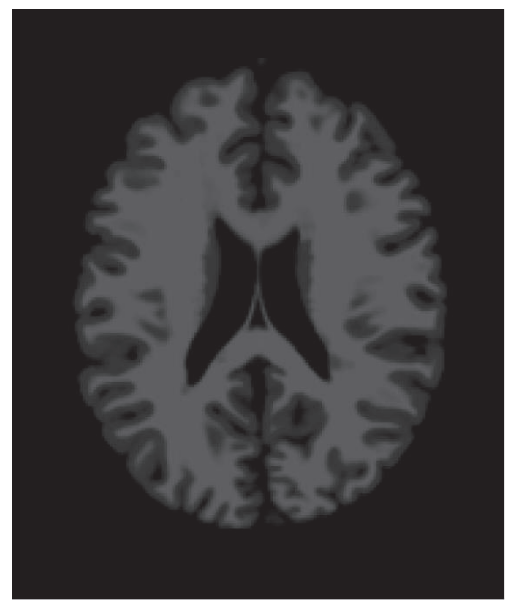

(d)

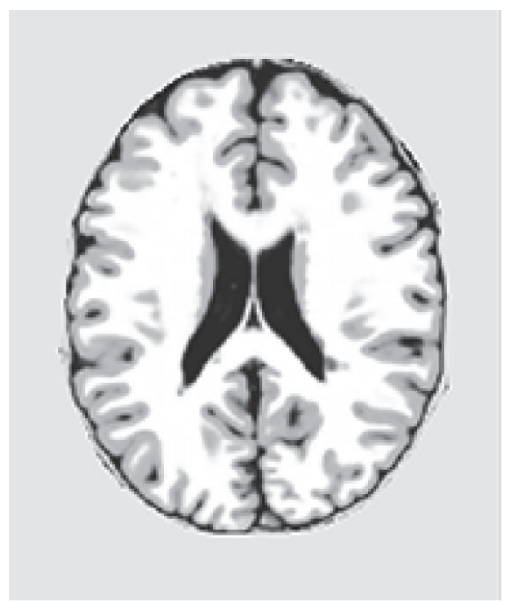

(g)

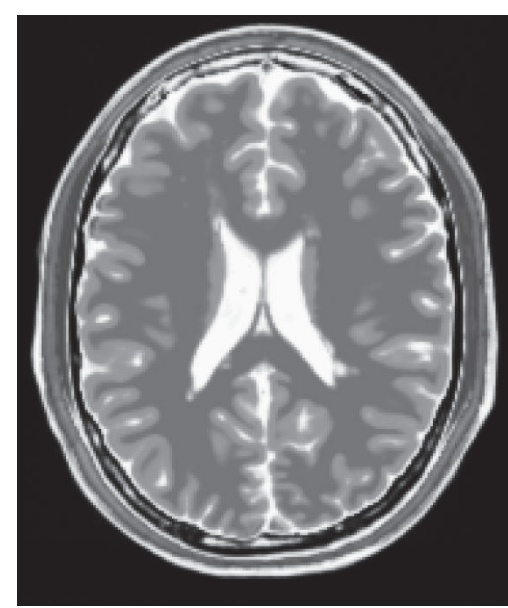

(b)

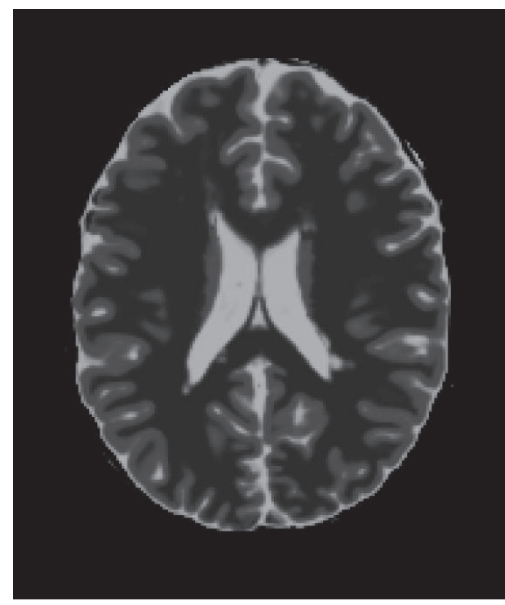

(e)

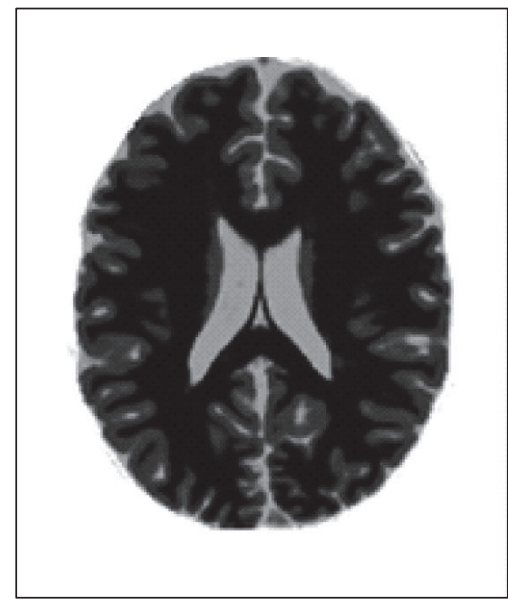

(h)

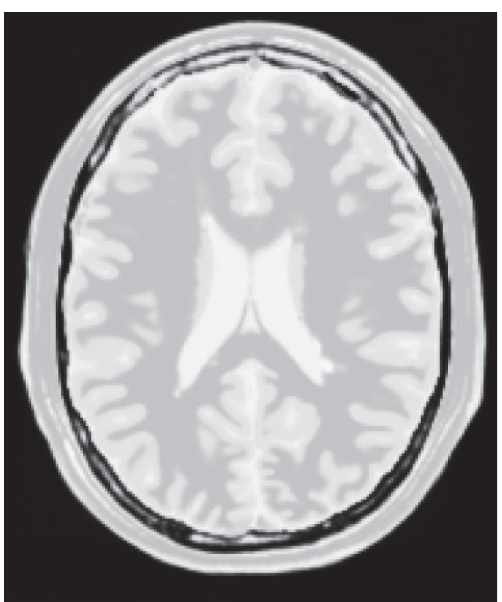

(c)

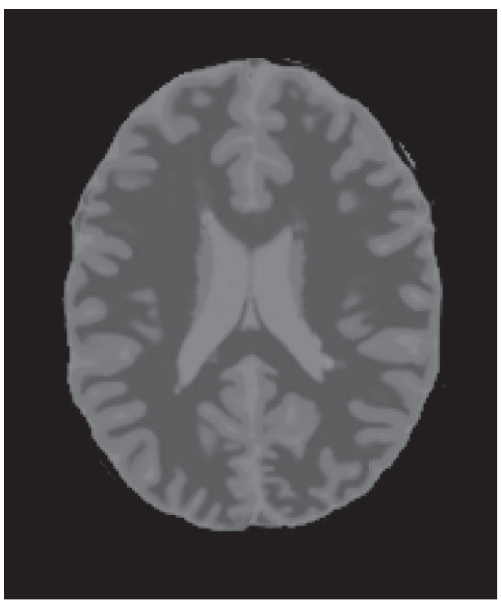

(f)

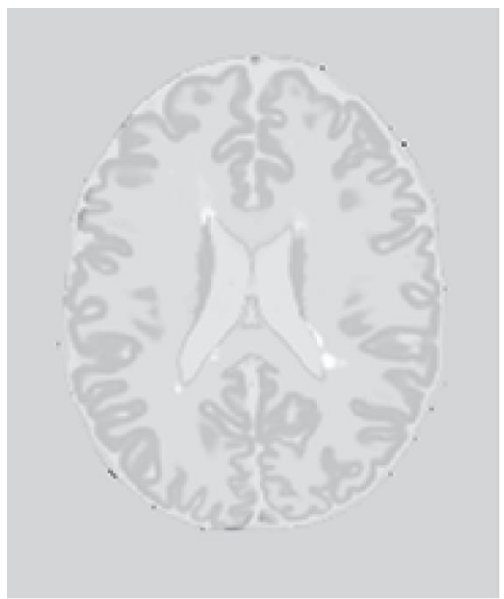

(i)

FIGURE 4: Extracted independent components by FastICA algorithm. ((a) to (c)) Original T1-weighted, T2-weighted, and PD-weighted images. ((d) to (f)) Results of images without skull region after centering and whitening. ((g) to (i)) Three independent components are extracted by FastICA algorithm. 


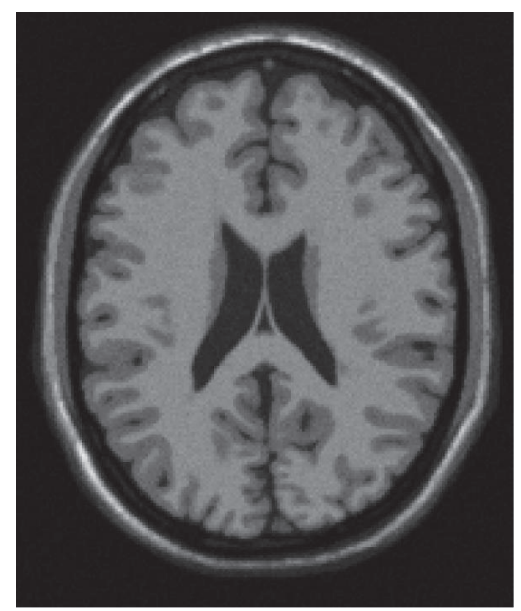

(a)

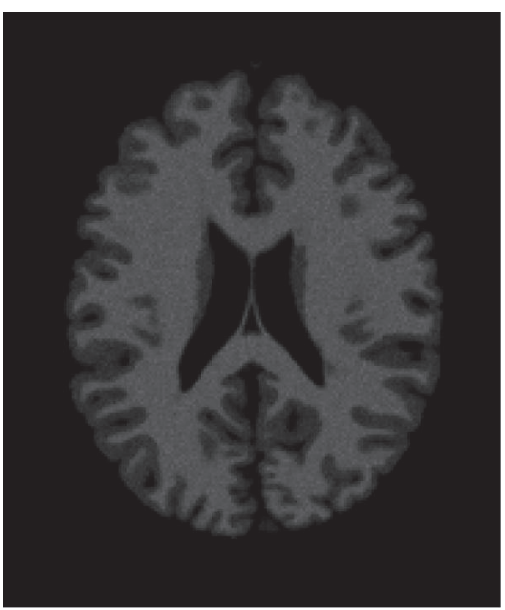

(d)

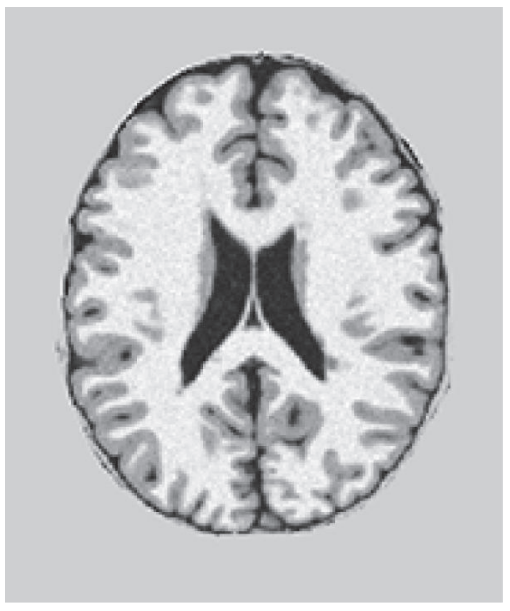

(g)

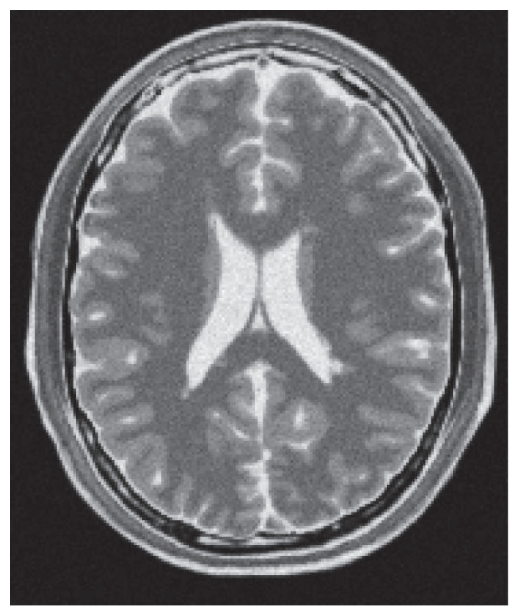

(b)

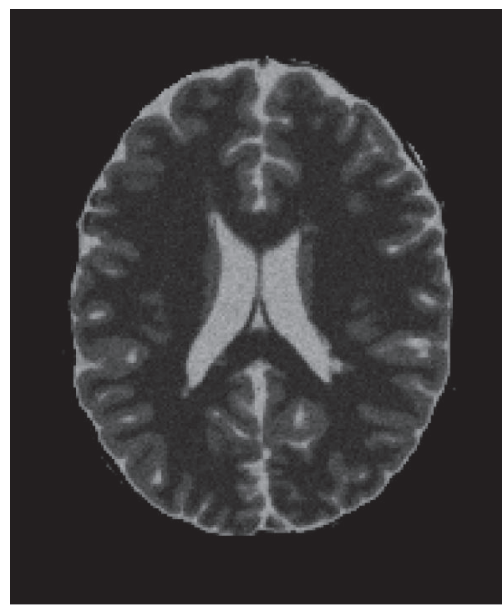

(e)

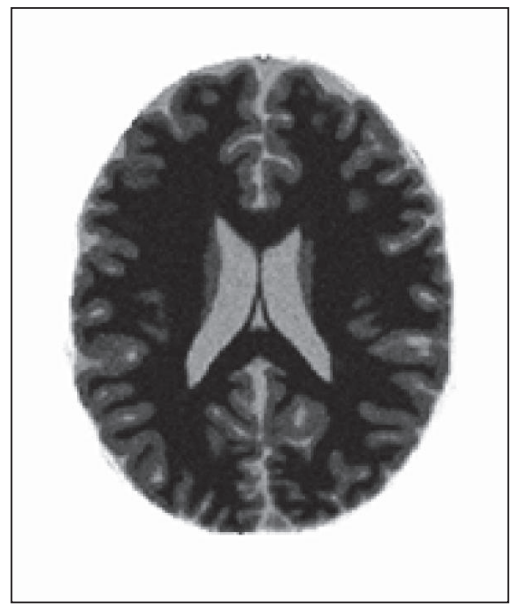

(h)

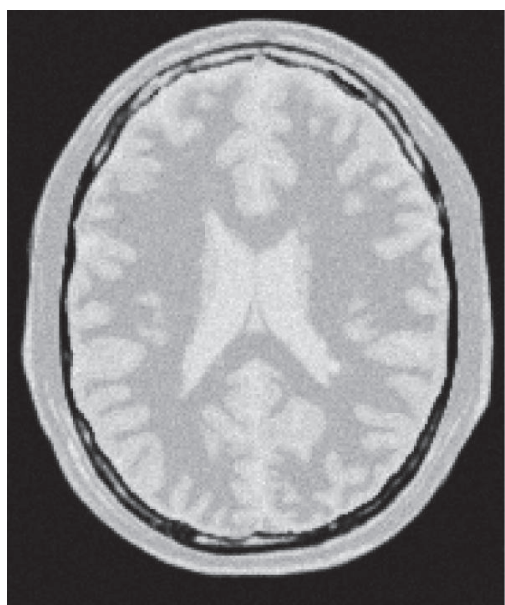

(c)

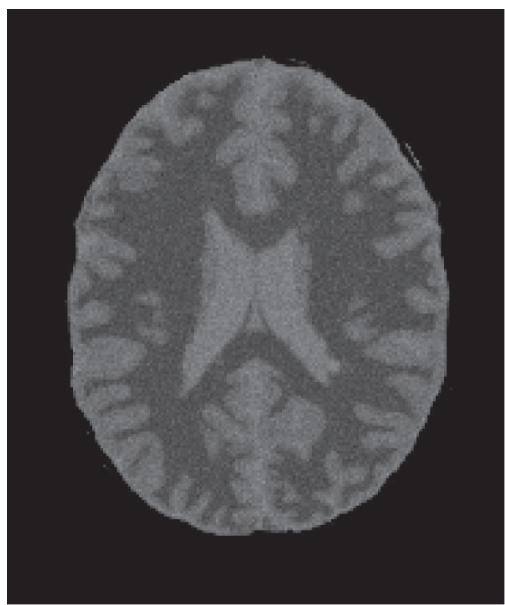

(f)

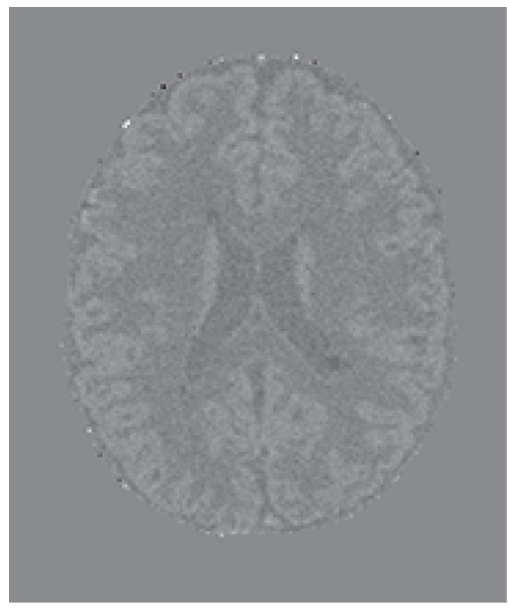

(i)

FIGURE 5: Extracted independent components by FastICA algorithm when the images are corrupted by 3\% noise. ((a) to (c)) Original T1weighted, T2-weighted, and $P D$-weighted images. ((d) to (f)) Results of images without skull region after centering and whitening. ((g) to (i)) Three independent components are extracted by FastICA algorithm. 


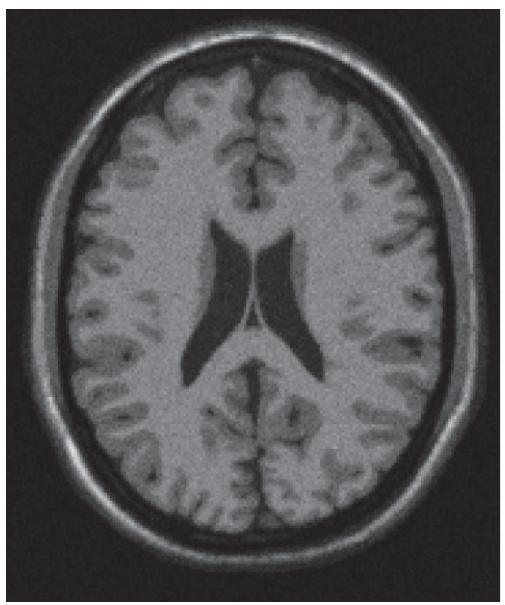

(a)

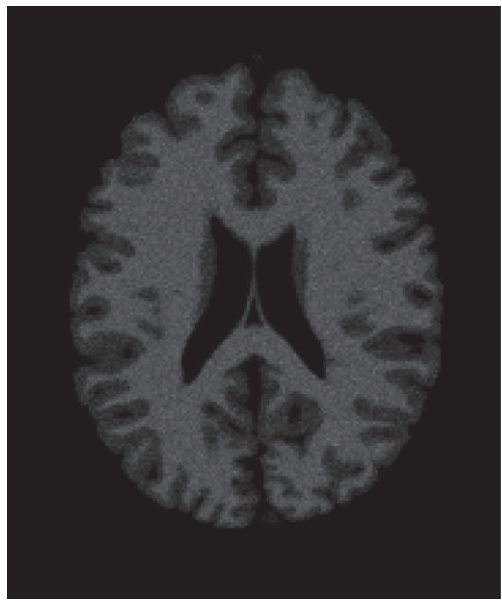

(d)

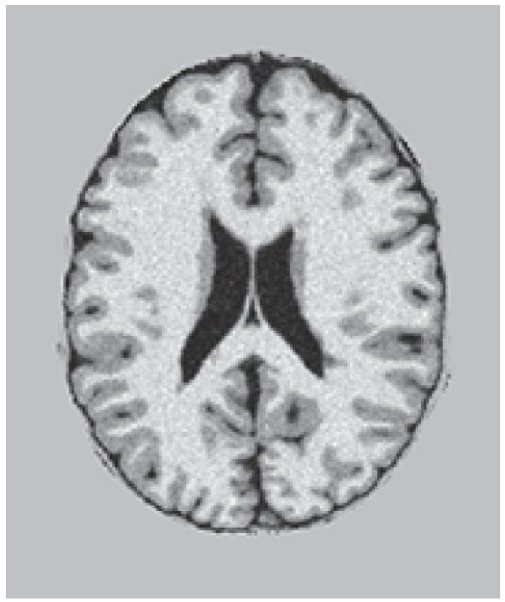

(g)

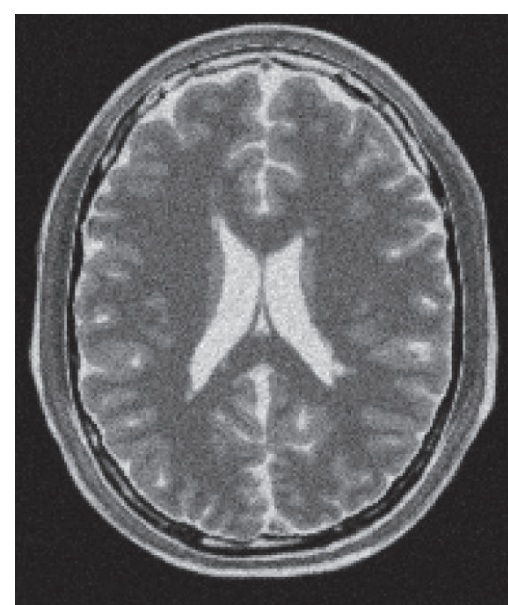

(b)

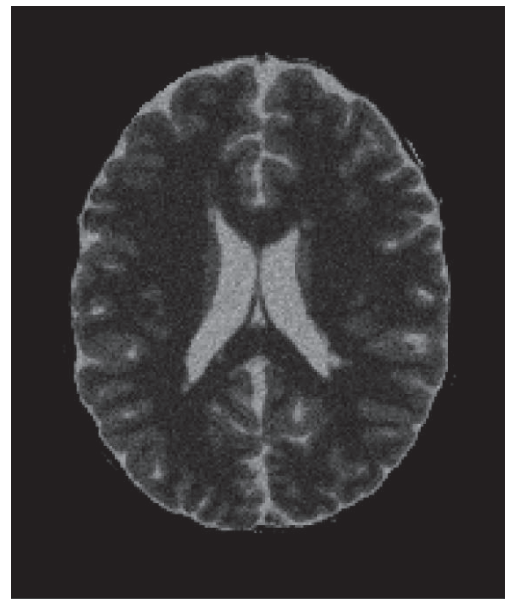

(e)

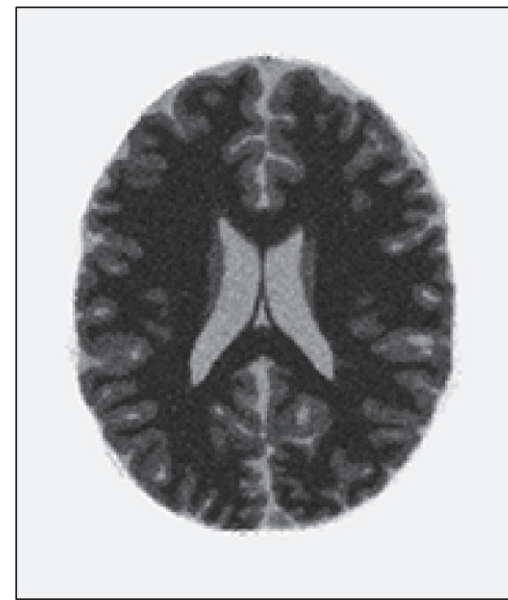

(h)

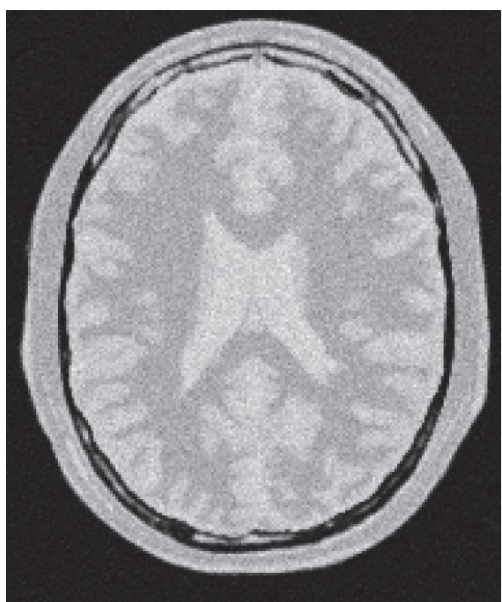

(c)

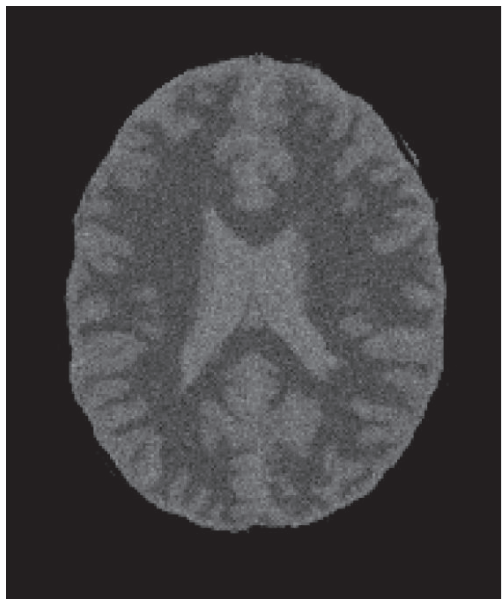

(f)

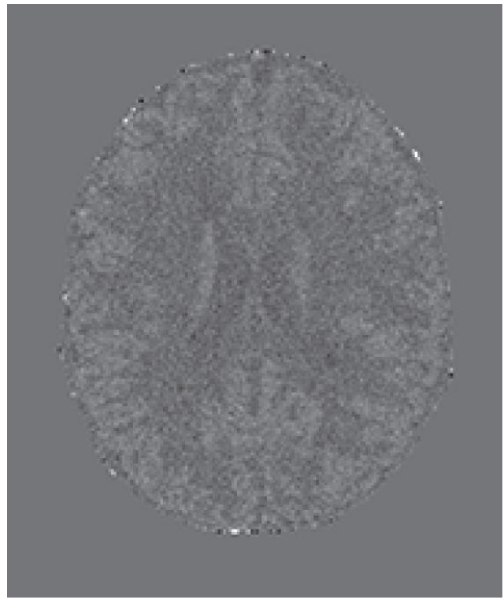

(i)

FIGURE 6: Extracted independent components by FastICA algorithm when the images are corrupted by 5\% noise. ((a) to (c)) Original T1weighted, T2-weighted, and $P D$-weighted images. ((d) to (f)) Results of images without skull region after centering and whitening. ((g) to (i)) Three independent components are extracted by FastICA algorithm. 


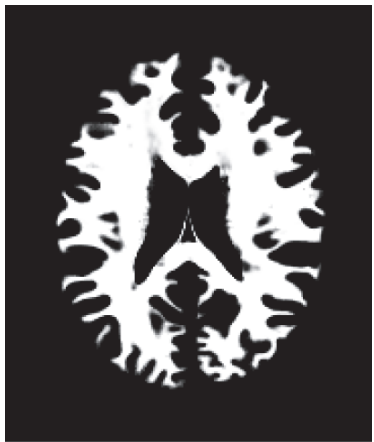

(a)

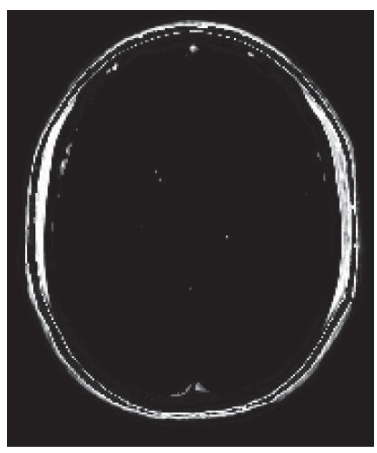

(d)

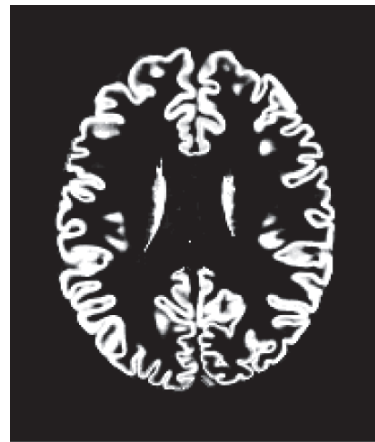

(b)

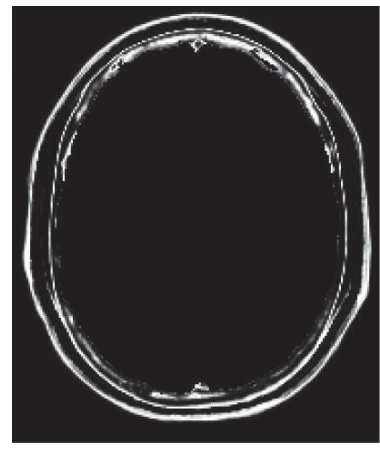

(e)

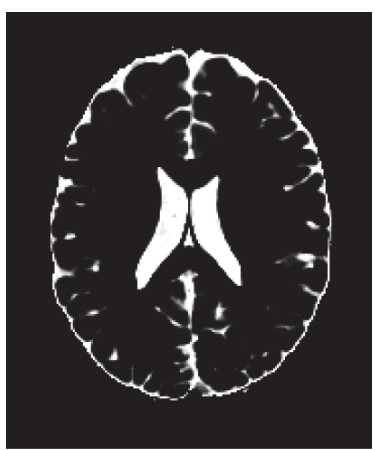

(c)

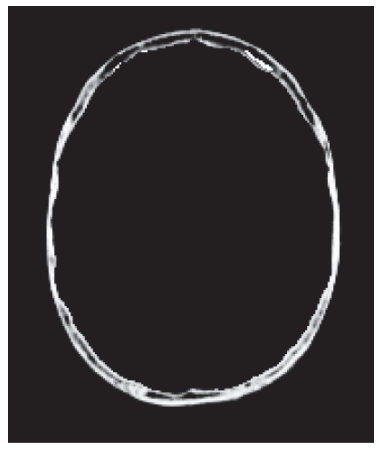

(f)

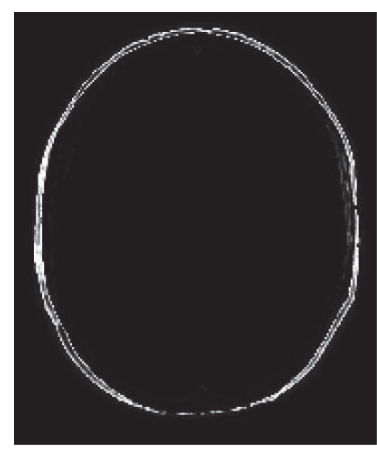

(g)

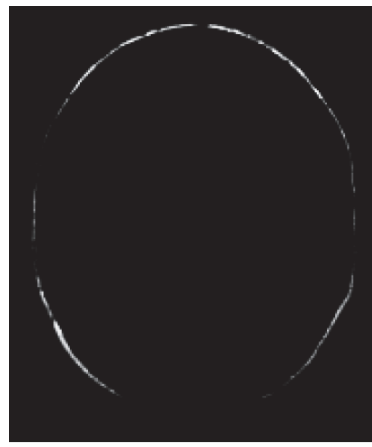

(h)

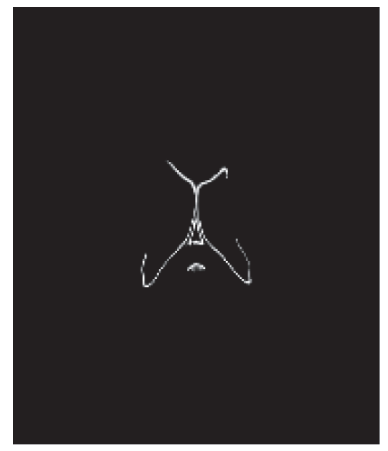

(i)

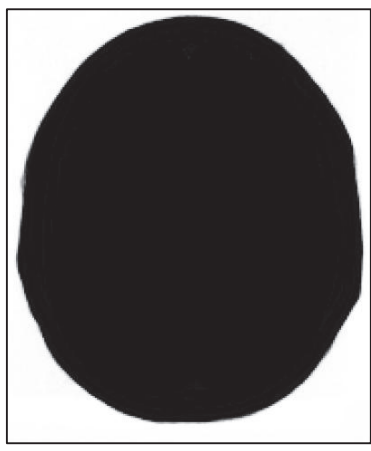

(j)

FIGURE 7: Ground truth of the brain tissue of simulated MRI image of the 94th slice. (a) WM. (b) GM. (c) CSF. (d) Muscle/skin. (e) Skin. (f) Skull. (g) Connective tissue. (h) Fat. (i) Glial matter. (j) Background.

coefficient indicates a better match between the segmented region and the ground truth.

The results of the corresponding measured similarity for Figures 8-10 using the six methods with three classes are shown in Tables $1-3$, and the level of noise varies from 0 to $5 \%$. Note that the noise was calculated relative to the brightest tissue with image size $=181 \times 217$, slice thickness $=1 \mathrm{~mm}$, and intensity nonuniformity ("RF") with $0 \%$. In all cases with different noise levels, the $k$-means method failed to correctly classify the brain tissue, demonstrating the worst performance especially for GM segmentation with a Jaccard coefficient lower than 0.13 . As ICA analysis can be used for contrast enhancement of MRI images, the two methods (ICFCM and ICKFCM) based on ICA signals decomposition are assumed to be able to achieve better performance than a method without ICA analysis. The assumption was confirmed by the quantitative evaluation of the experiments as the Jaccard coefficients evaluated by ICFCM and ICKFCM appeared to be greater than those of FCM, KFCM, and KWFLICM. Moreover, in all tables, it can be observed that the proposed ICKFCM algorithm reports the largest Jaccard coefficient among the six methods; that is, the proposed method delivers the best segmentation result.

To validate the suitability of the proposed method for segmentation of multimodal $M R I$ images, we also examined the ability of brain tissue extraction when the original images were degraded by several processes in terms of $3 \times 3$ averaging, Gaussian smoothing of $\mu=5$ and $\sigma=5$, and adding 


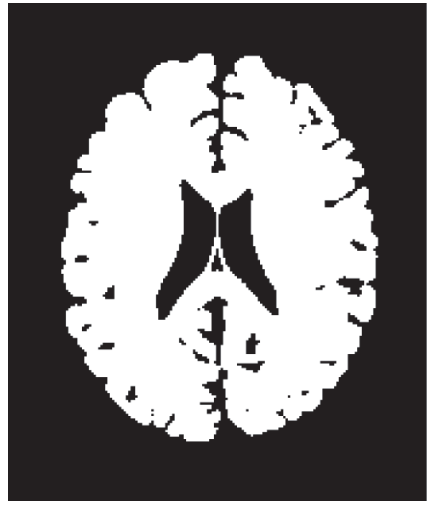

(a)

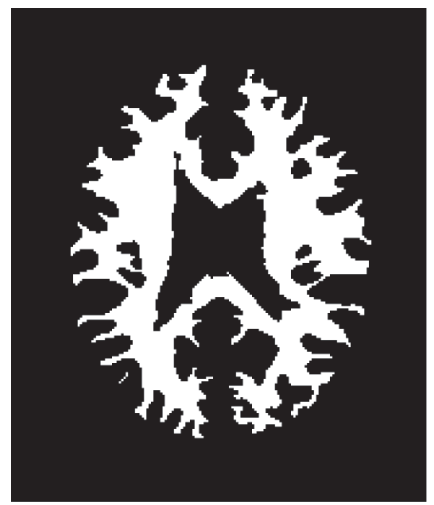

(d)

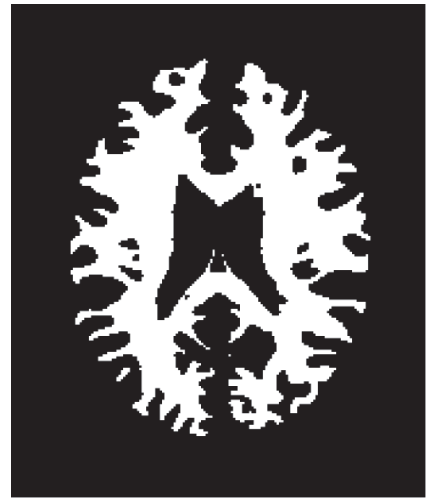

(g)

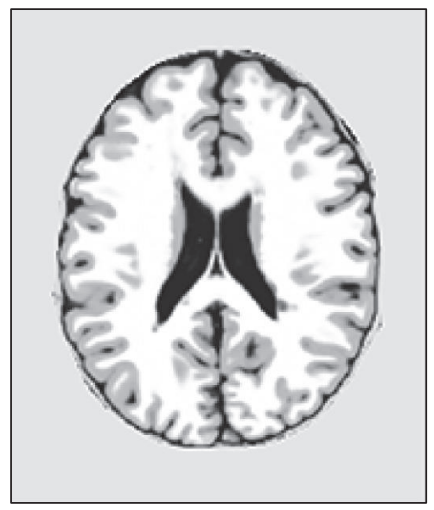

(j)

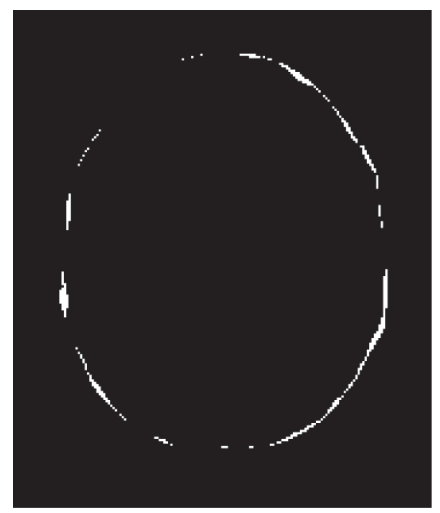

(b)

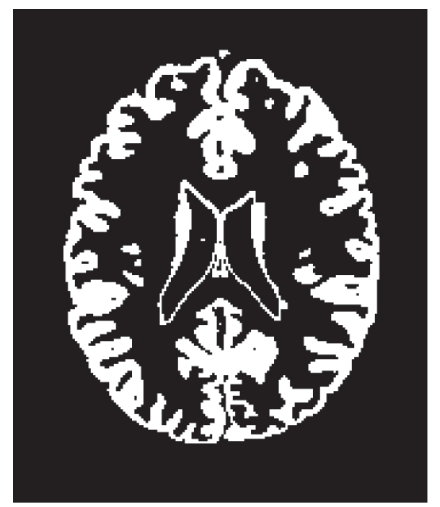

(e)

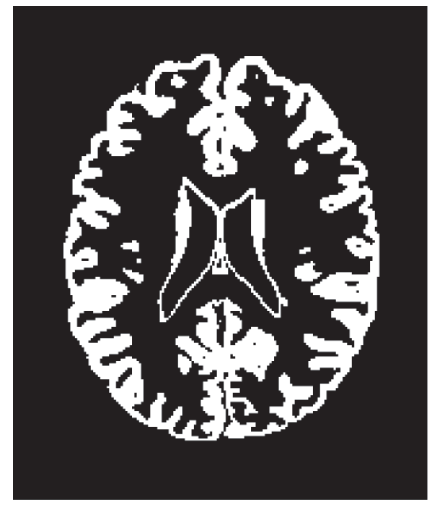

(h)

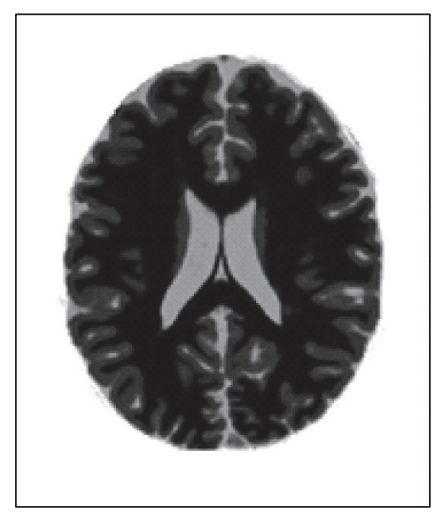

(k)

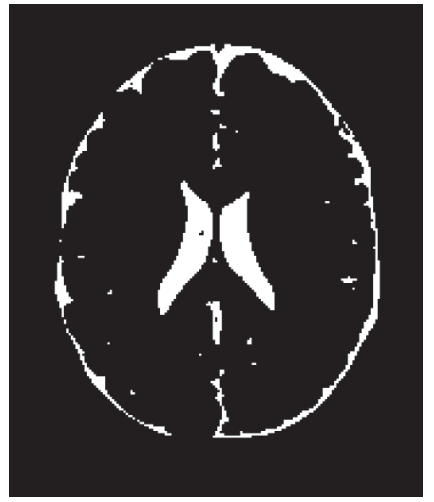

(c)

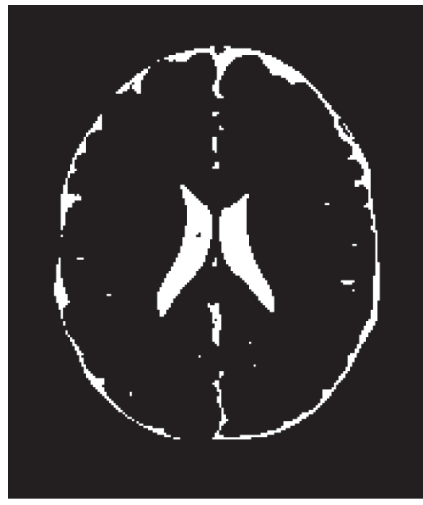

(f)

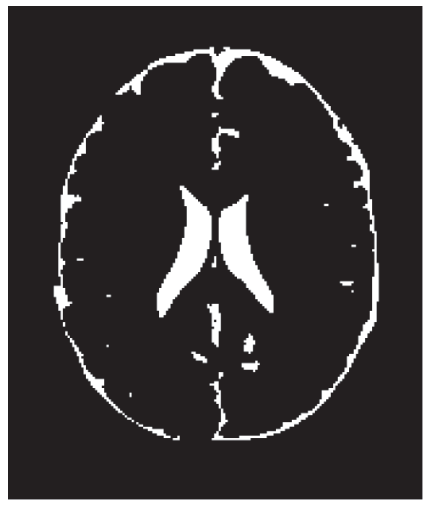

(i)

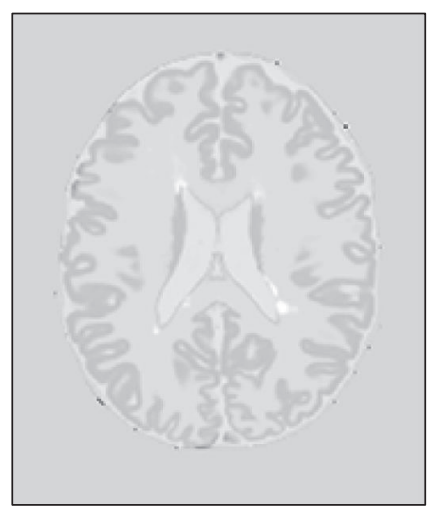

(l)

FIgURE 8: Continued. 


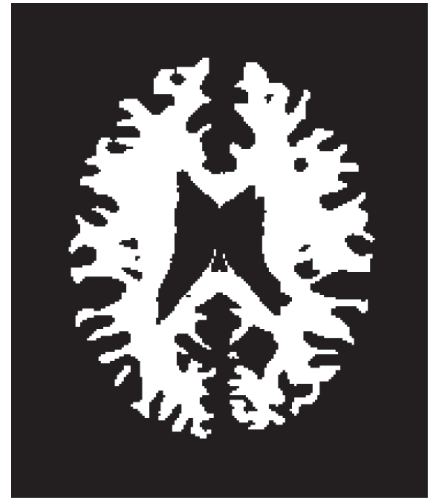

(m)

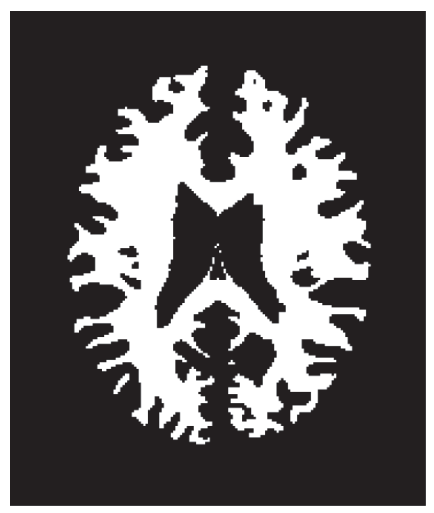

(p)

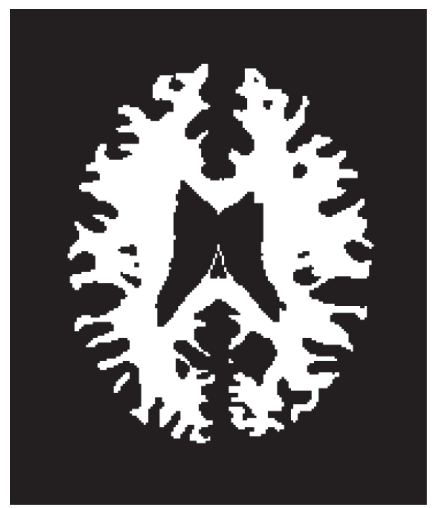

(s)

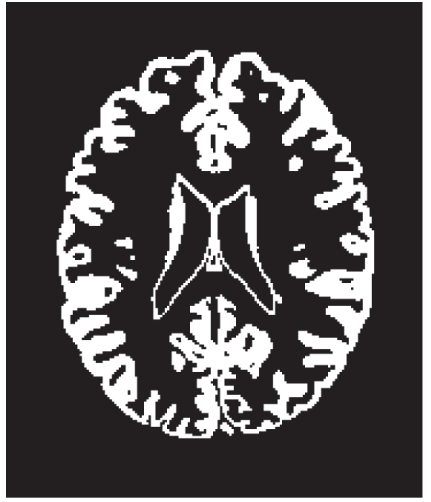

(n)

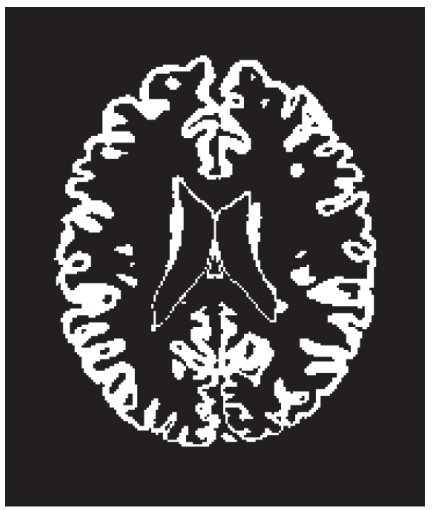

(q)

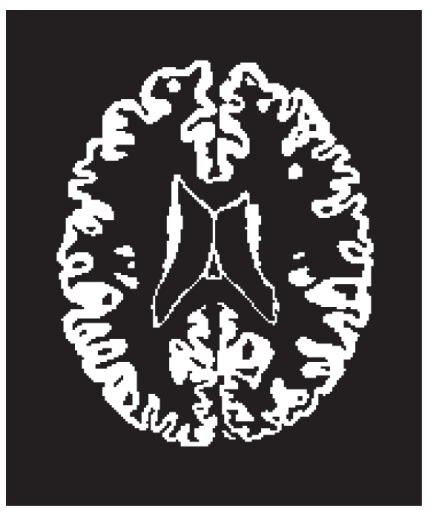

(t)

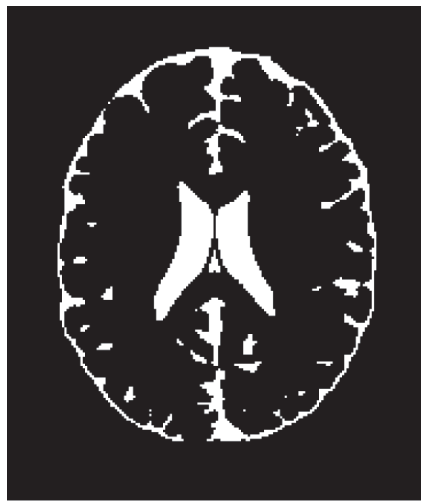

(o)

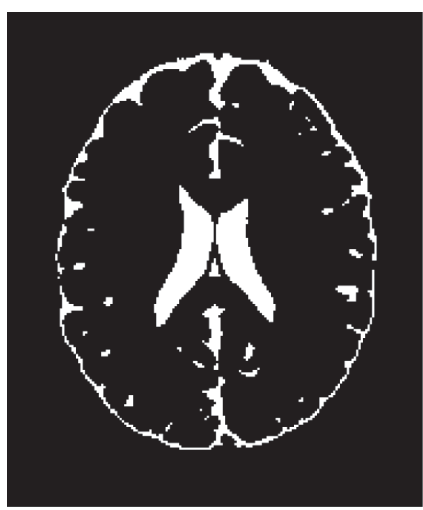

$(\mathrm{r})$

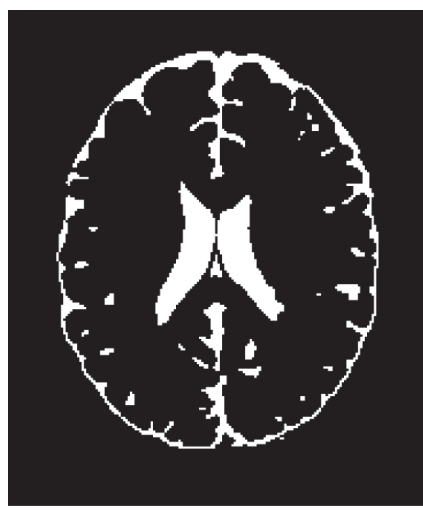

(u)

FIGURE 8: Segmentation comparisons when simulated MRI images do not suffer noise corruption. ((a) to (c)) Segmented results of WM, GM, and CSF + glial matter using $k$-means algorithm. ((d) to (f)) Using FCM. ((g) to (i)) Using KFCM. ((j) to (l)) The three extracted independent components using FastICA. ((m) to (o)) Using KWFLICM. ((p) to (r)) Using ICFCM. ((s) to (u)) Using ICKFCM.

TABLE 1: Measured similarity for noiseless case (Figure 8) using six methods with three classes.

\begin{tabular}{|c|c|c|c|c|c|c|}
\hline \multirow{2}{*}{ Tissues } & \multicolumn{6}{|c|}{ Methods } \\
\hline & $k$-means & FCM & KFCM & KWFLICM & ICFCM & ICKFCM \\
\hline$W M$ & 0.3513 & 0.6379 & 0.6311 & 0.8305 & 0.8303 & 0.9116 \\
\hline$G M$ & 0.2744 & 0.5779 & 0.6938 & 0.8507 & 0.8454 & 0.8543 \\
\hline CSF + glial matter & 0.6845 & 0.7241 & 0.7283 & 0.7244 & 0.7976 & 0.8350 \\
\hline Average & 0.4367 & 0.6466 & 0.6844 & 0.8019 & 0.8244 & 0.8607 \\
\hline
\end{tabular}




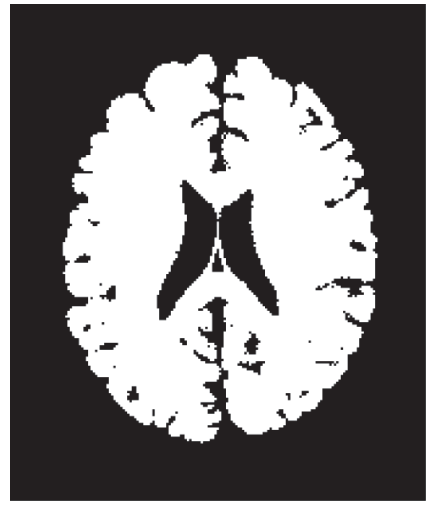

(a)

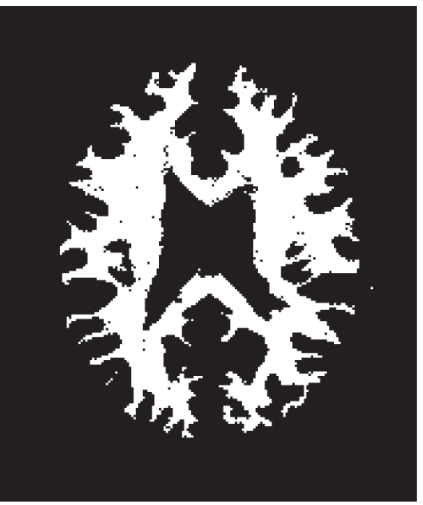

(d)

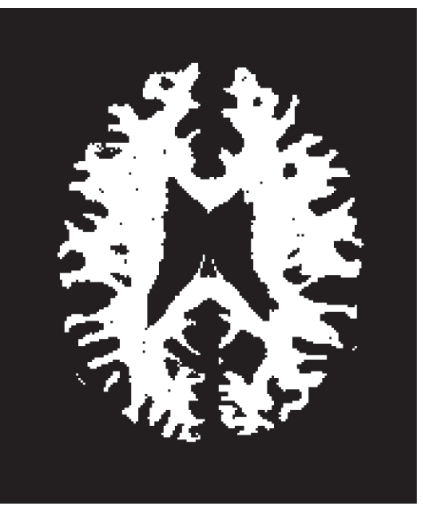

(g)

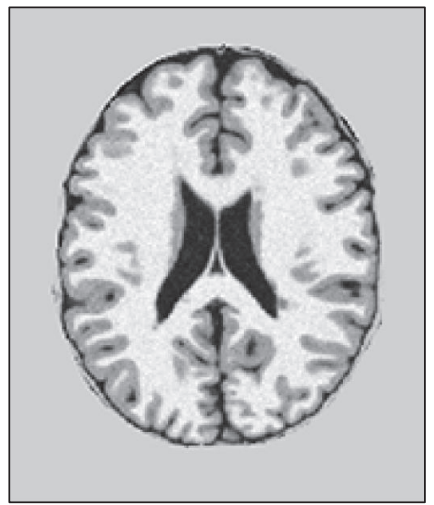

(j)

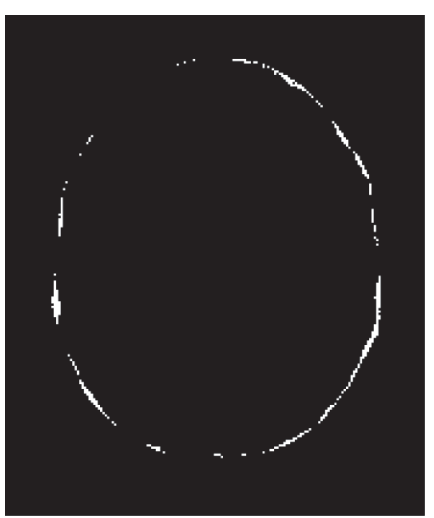

(b)

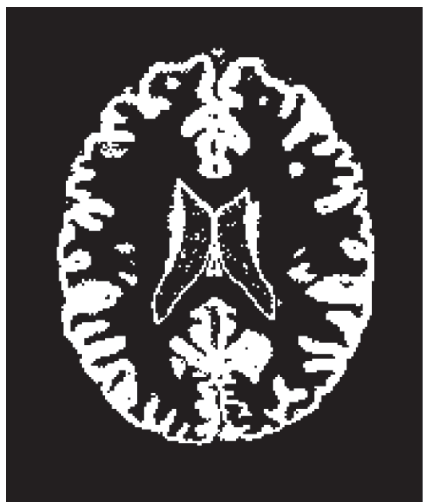

(e)

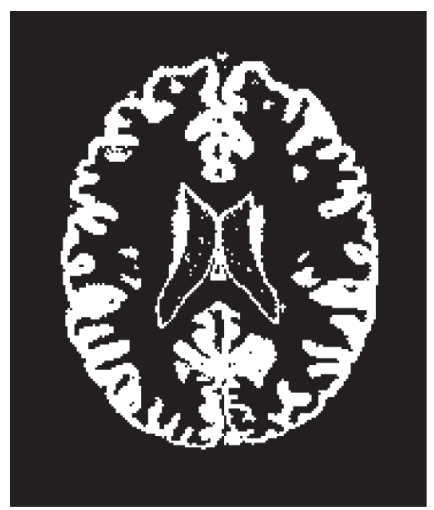

(h)

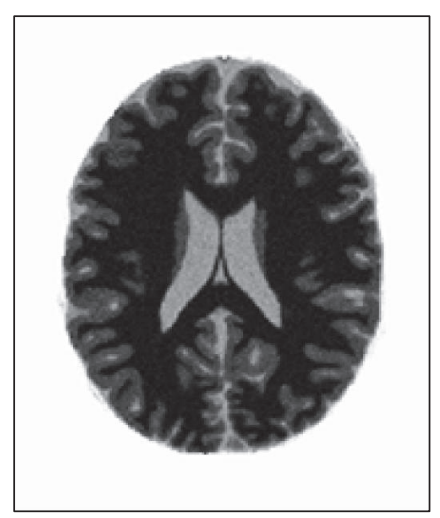

(k)

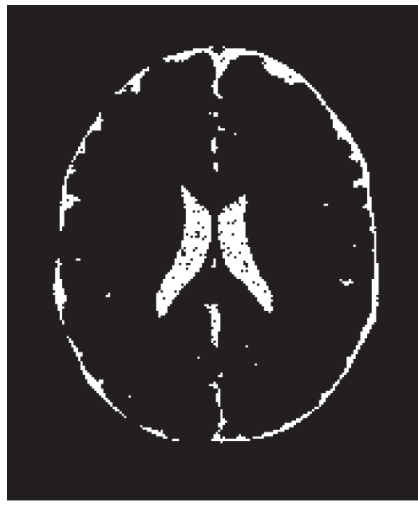

(c)

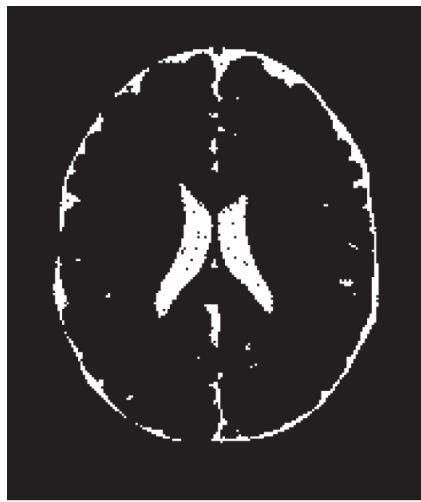

(f)

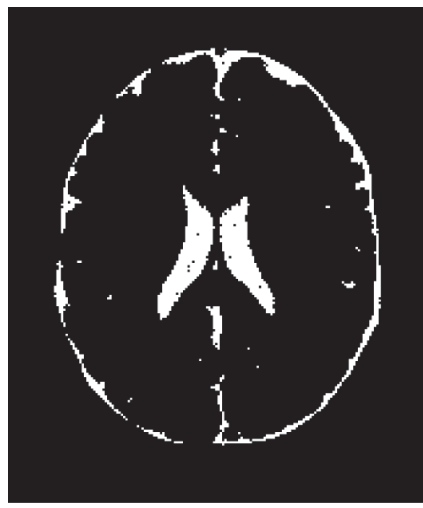

(i)

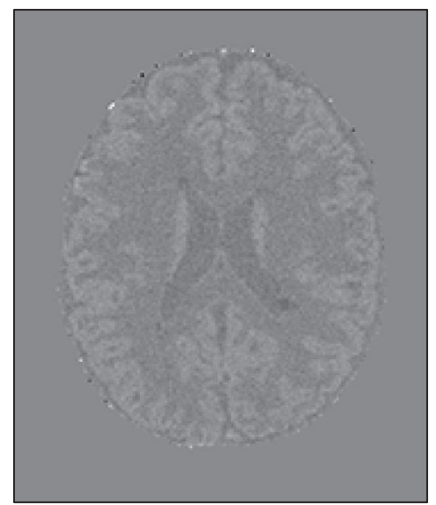

(1)

FIgURe 9: Continued. 


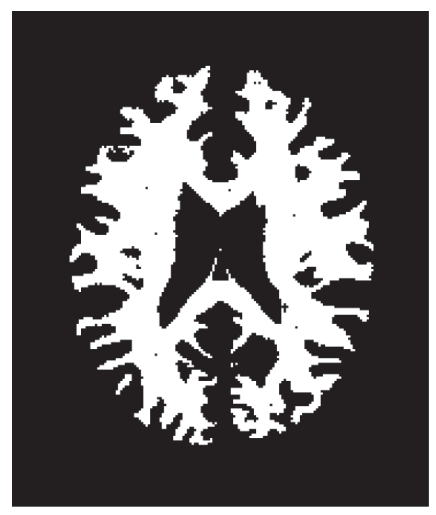

(m)

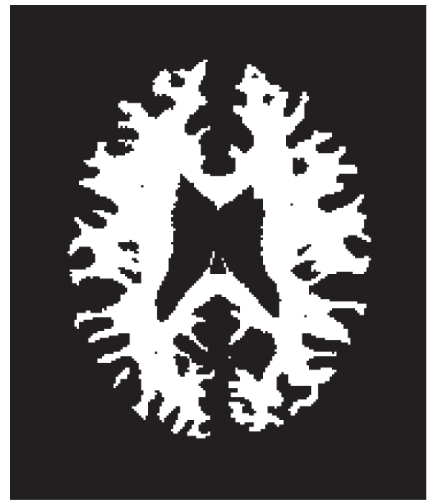

(p)

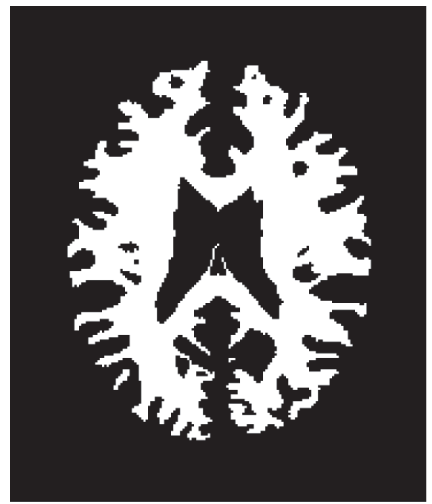

(s)

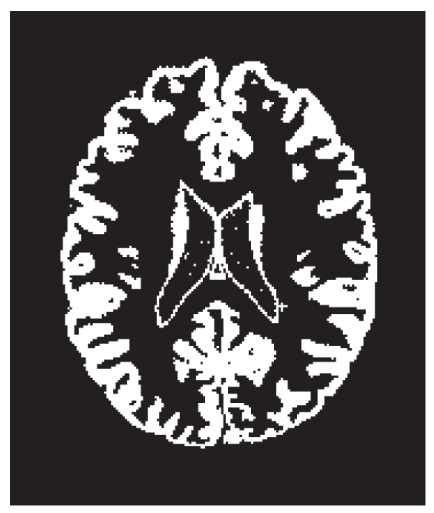

(n)

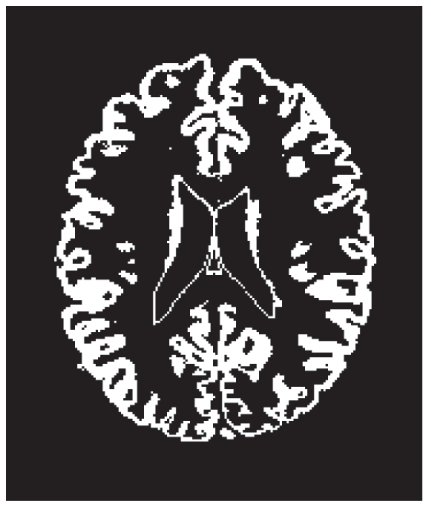

(q)

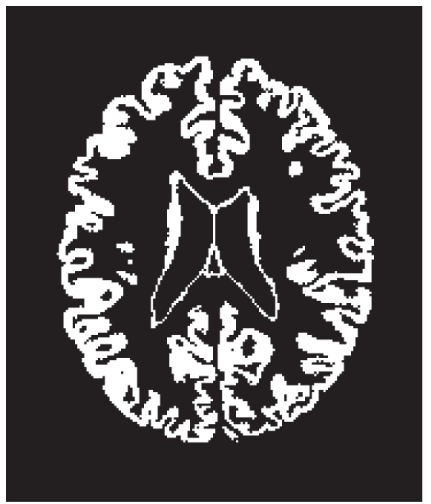

$(\mathrm{t})$

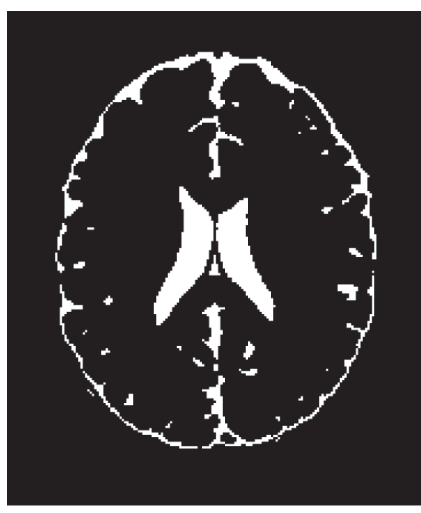

(o)

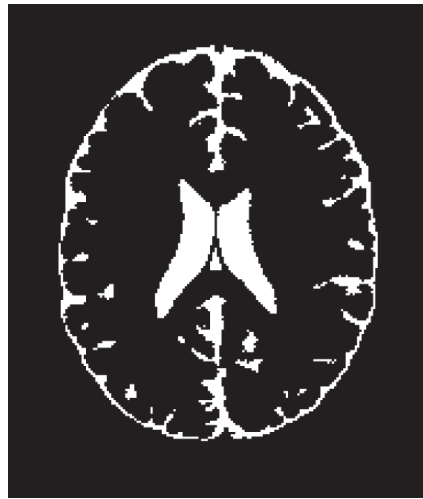

$(\mathrm{r})$

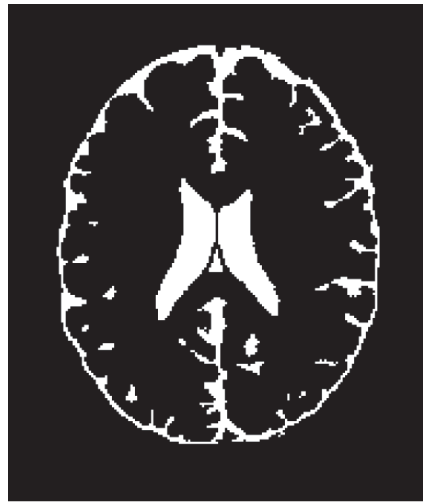

(u)

FiguRE 9: Segmentation comparisons when simulated MRI images have 3\% noise. ((a) to (c)) Segmented results of WM, GM, and CSF + glial matter using $k$-means algorithm. ((d) to (f)) Using FCM. ((g) to (i)) Using KFCM. ((j) to (l)) The three extracted independent components using FastICA. ((m) to (o)) Using KWFLICM. ((p) to (r)) Using ICFCM. ((s) to (u)) Using ICKFCM.

TABLE 2: Measured similarity for 3\% noise (Figure 9) using six methods with three classes.

\begin{tabular}{|c|c|c|c|c|c|c|}
\hline \multirow{2}{*}{ Tissues } & \multicolumn{6}{|c|}{ Methods } \\
\hline & $k$-means & FCM & KFCM & KWFLICM & ICFCM & ICKFCM \\
\hline$W M$ & 0.3304 & 0.6008 & 0.6233 & 0.8496 & 0.8142 & 0.8572 \\
\hline$G M$ & 0.1841 & 0.5110 & 0.6392 & 0.6935 & 0.6937 & 0.7146 \\
\hline$C S F+$ glial matter & 0.6237 & 0.6462 & 0.7149 & 0.7707 & 0.7753 & 0.8232 \\
\hline Average & 0.3794 & 0.5860 & 0.6590 & 0.7713 & 0.7611 & 0.7983 \\
\hline
\end{tabular}




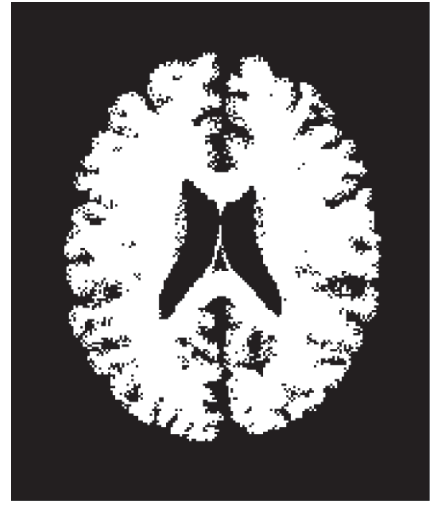

(a)

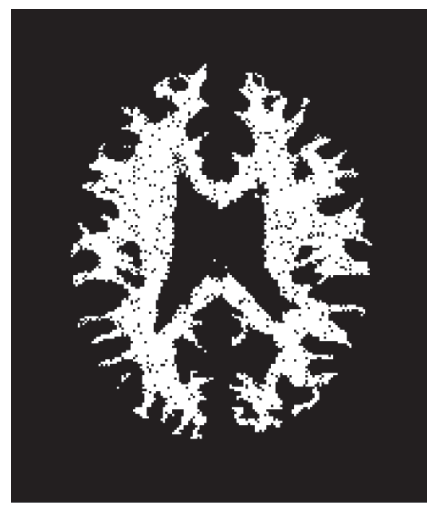

(d)

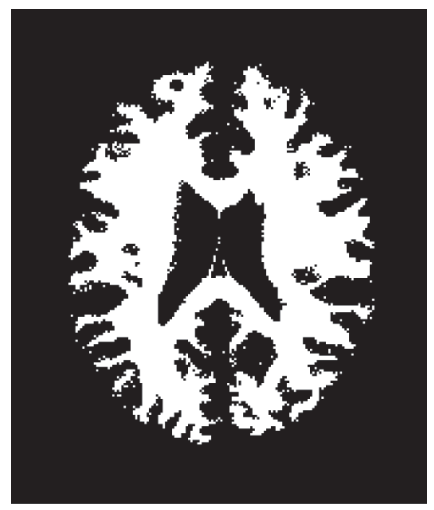

(g)

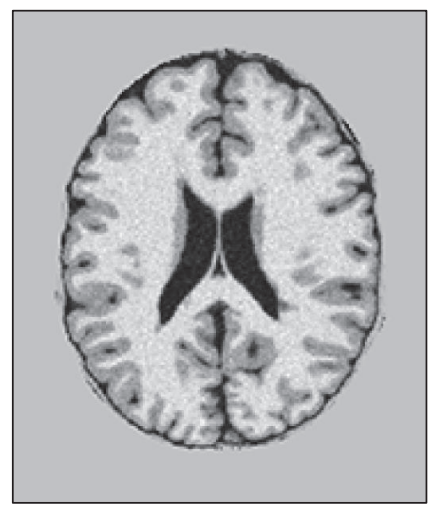

(j)

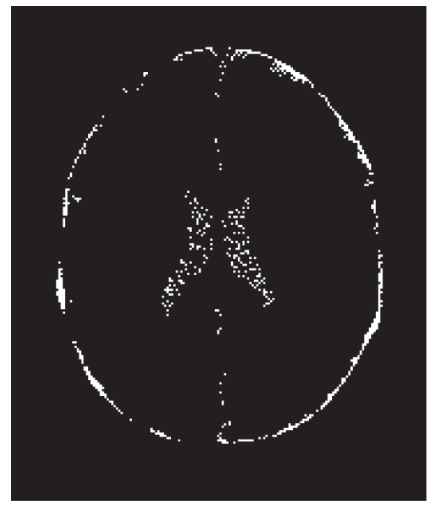

(b)

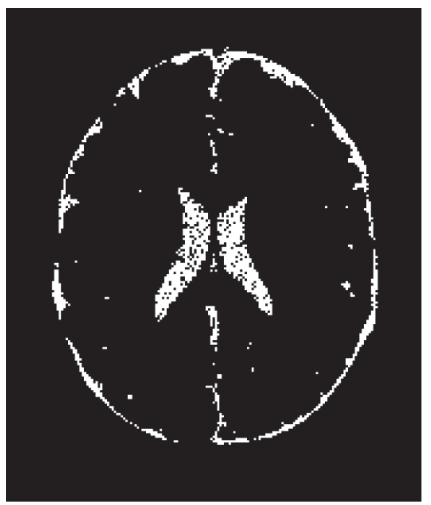

(e)

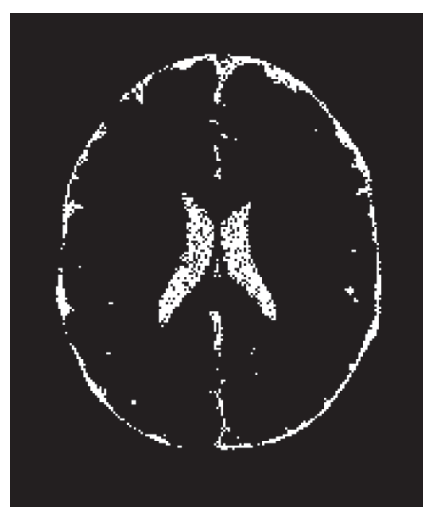

(h)

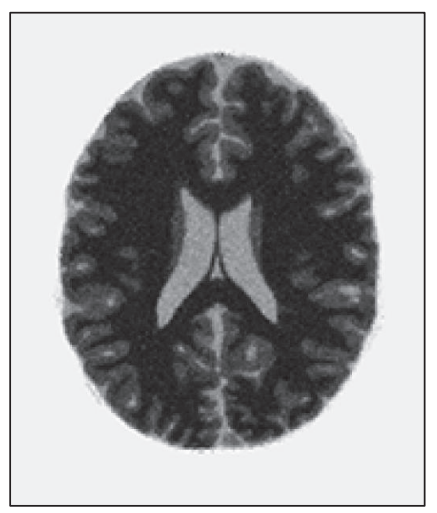

(k)

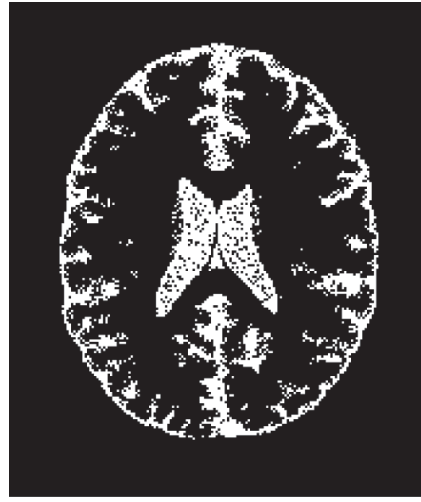

(c)

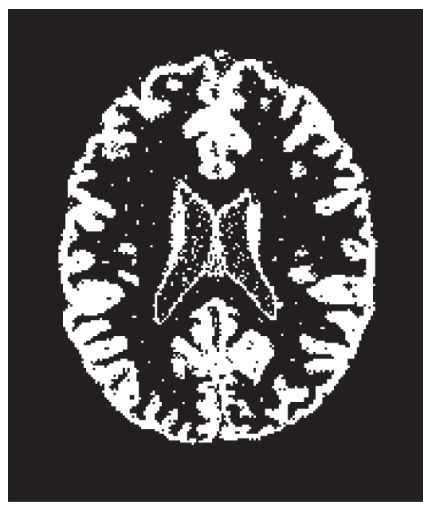

(f)

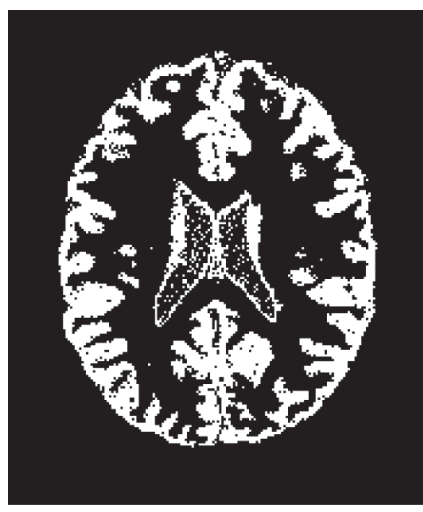

(i)

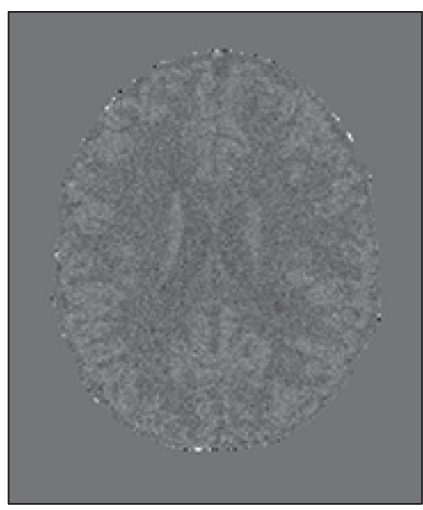

(l)

Figure 10: Continued. 


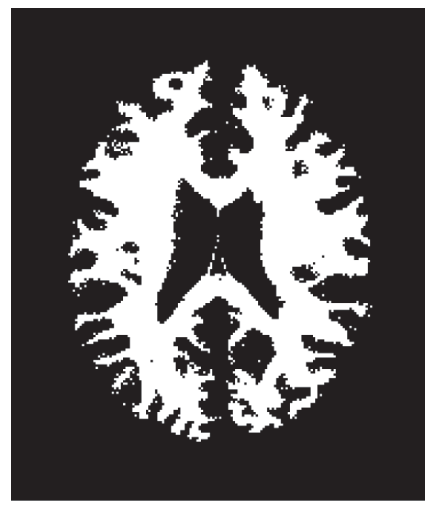

(m)

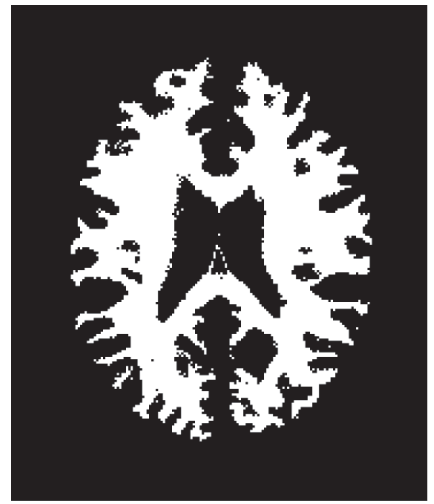

(p)

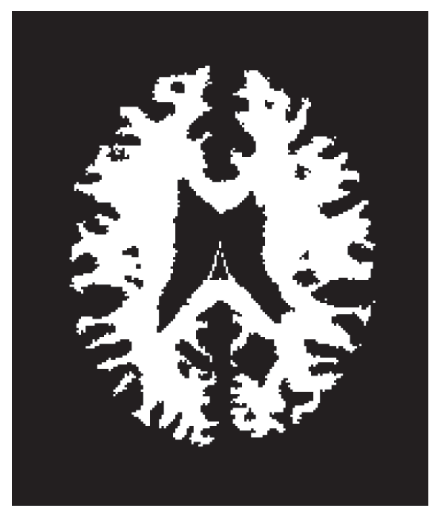

(s)

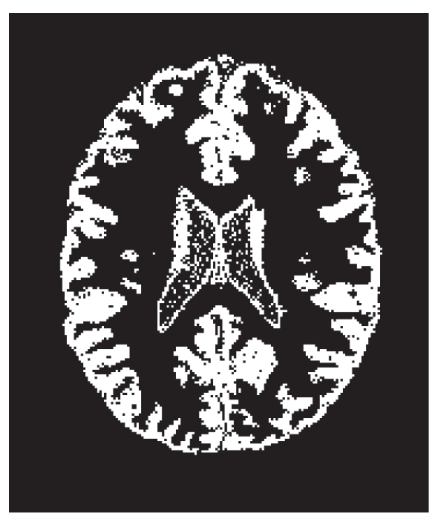

(n)

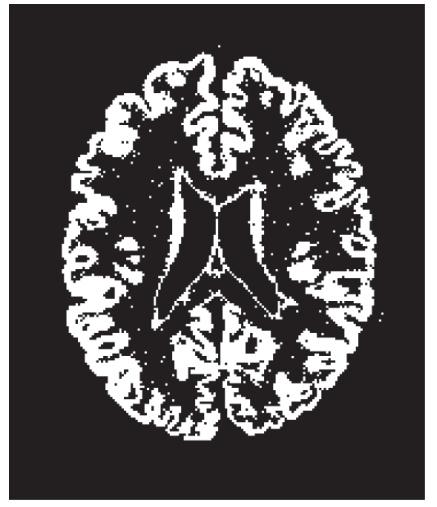

(q)

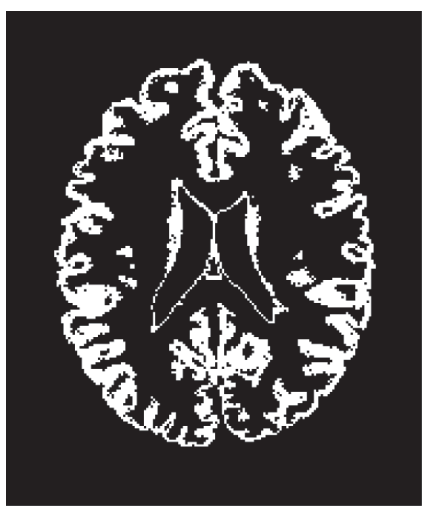

(t)

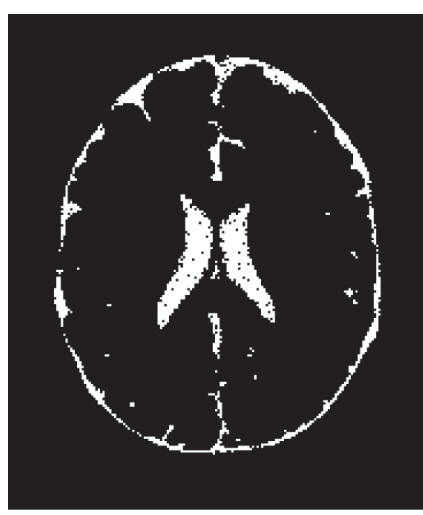

(o)

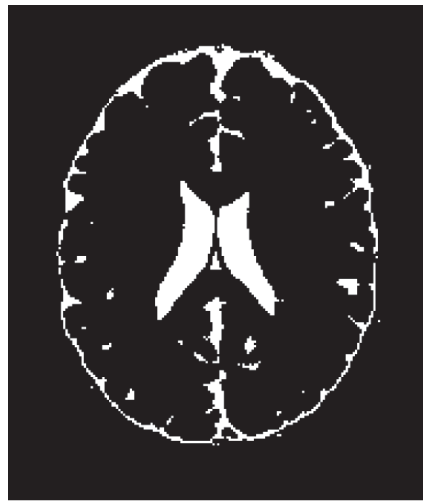

(r)

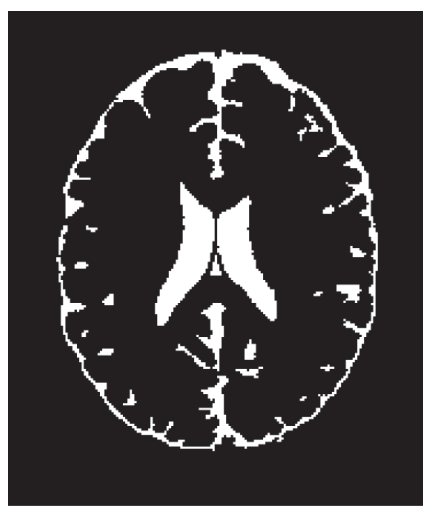

(u)

FIGURE 10: Segmentation comparisons when simulated MRI images have 5\% noise. ((a) to (c)) Segmented results of WM, GM, and CSF + glial matter using $k$-means algorithm. ((d) to (f)) Using FCM. ((g) to (i)) Using KFCM. ((j) to (l)) The three extracted independent components using FastICA. ((m) to (o)) Using KWFLICM. ((p) to (r)) Using ICFCM. ((s) to (u)) Using ICKFCM.

TABLE 3: Measured similarity for 5\% noise (Figure 10) using six methods with three classes.

\begin{tabular}{|c|c|c|c|c|c|c|}
\hline \multirow{2}{*}{ Tissues } & \multicolumn{6}{|c|}{ Methods } \\
\hline & $k$-means & $F C M$ & KFCM & KWFLICM & ICFCM & ICKFCM \\
\hline$W M$ & 0.2928 & 0.5738 & 0.5499 & 0.5715 & 0.6013 & 0.6482 \\
\hline$G M$ & 0.1234 & 0.4532 & 0.4732 & 0.5839 & 0.6092 & 0.6194 \\
\hline$C S F+$ glial matter & 0.4022 & 0.4970 & 0.6234 & 0.6844 & 0.7372 & 0.7589 \\
\hline Average & 0.2728 & 0.5080 & 0.5488 & 0.6133 & 0.6493 & 0.6755 \\
\hline
\end{tabular}




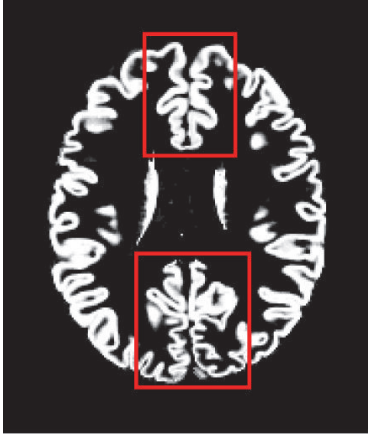

(a)

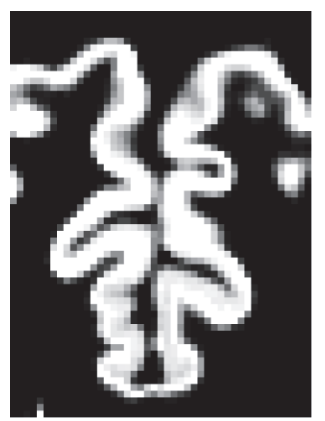

(e)

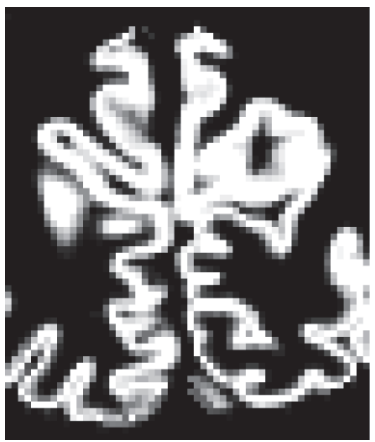

(i)

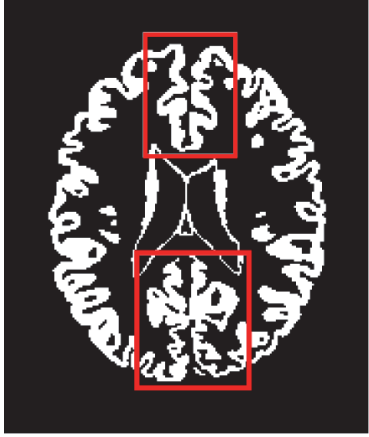

(b)

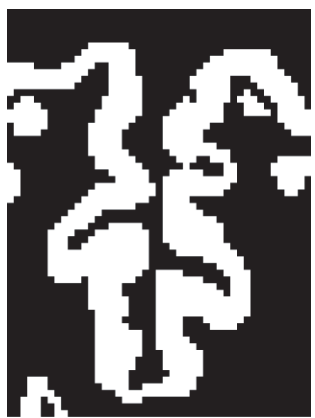

(f)

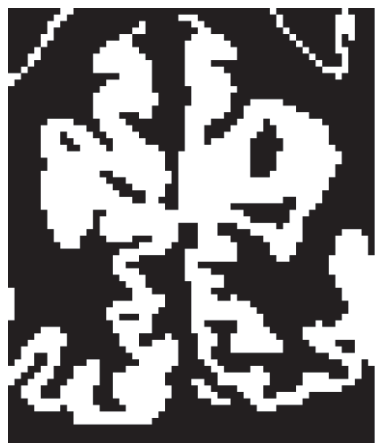

(j)

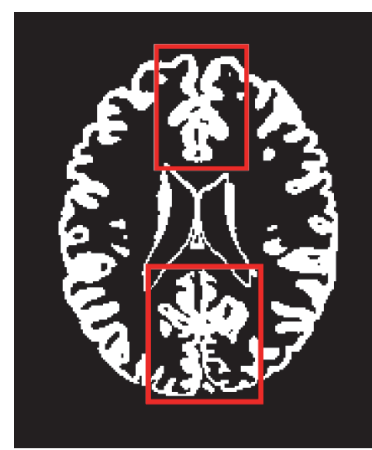

(c)

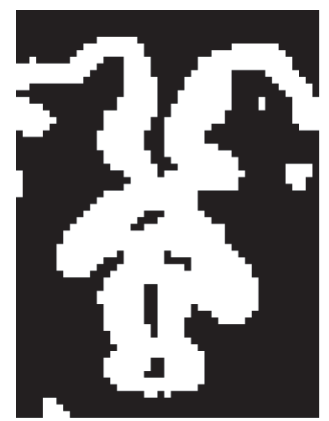

(g)

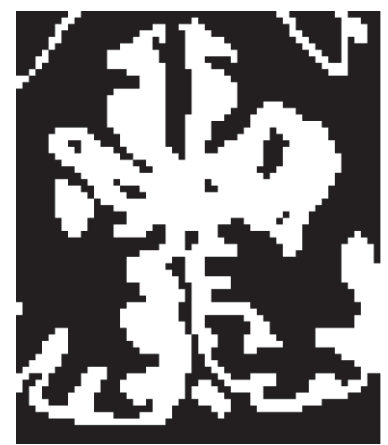

(k)

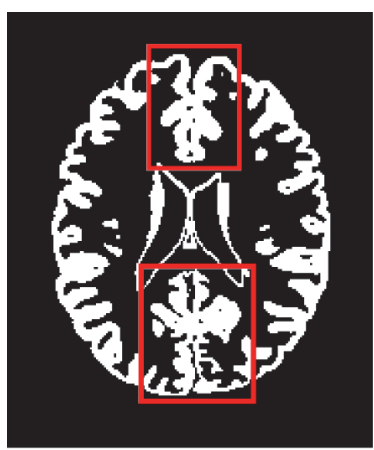

(d)

FIGURE 11: Visual comparison of segmentation results based on ICKFCM, KWFLICM, and KFCM algorithms, where red boxes denote the ROI for visual inspection and qualitative comparison. (a) The ground truth of GM. (b) The segmentation result of GM using ICKFCM algorithm. (c) Using KWFLICM algorithm. (d) Using KFCM algorithm. ((e) and (i)) The enlargement regions of ROI in ground truth of GM. ((f) and (j)) The enlargement regions of ROI in Figure 11(b). ((g) and (k)) In Figure 11(c). ((k) and (l)) In Figure 11(d).

$15 \%$ uniform random noise to the original images. Figures 12(a)-12(c) show the simulated $M R I$ images, T1-weighted, T2-weighted, and $P D$-weighted images of the 98th slice. The segmentation results of averaging operation, Gaussian smoothing, and adding uniform random noise are shown, respectively, in Figures 12(d)-12(f), Figures 12(g)-12(i), and Figures $12(\mathrm{j})-12(\mathrm{l})$. The ground truth, segmentation on noiseless original images, and skull region of the T1-weighted image are shown in Figures $12(\mathrm{~m})-12(\mathrm{t})$ for qualitative comparison. The quantitative performance of the proposed method was also calculated when the original images were corrupted by the processes mentioned above. As indicated in Table 4, the proposed method can extract the complicated shape of brain tissues and suppress various types of noise.

In addition to segmenting the simulated MRI images, the proposed method was further applied to real medical images as shown in Figures 13(a)-13(c). Figures 13(d)-13(f) show the images after skull stripping. Figures 13(g)-13(i) show the three independent components extracted by the FastICA algorithm. Figures $13(\mathrm{j})-13(\mathrm{~m})$ show that the skull region, $W M$, $G M$, and CSF plus glial matter were segmented by the proposed method. Finally, real MRI images, provided by the Yuanpei University of Medical Technology as shown in Figures 14(a)-14(c), were used to verify the performance of 


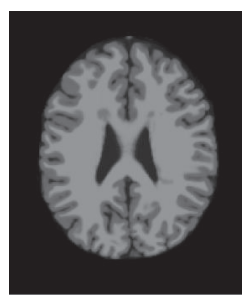

(a)

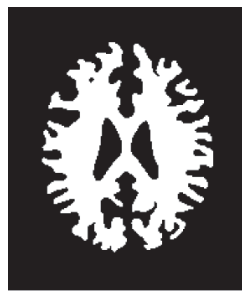

(g)

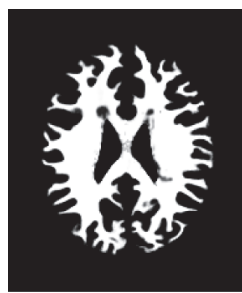

(m)

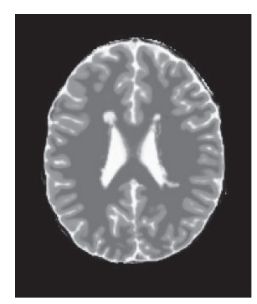

(b)

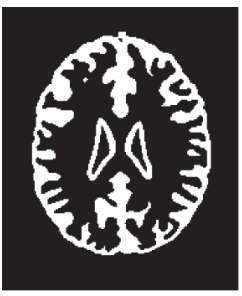

(h)

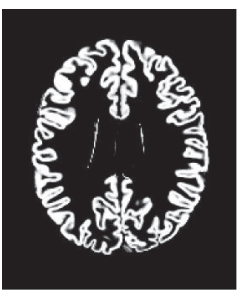

(n)

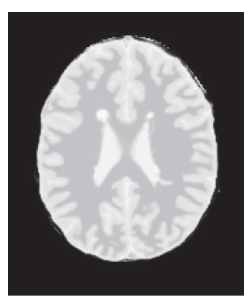

(c)

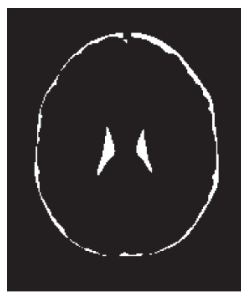

(i)

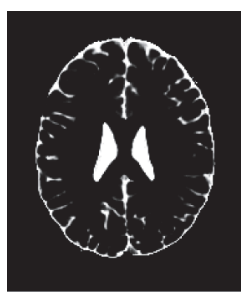

(o)

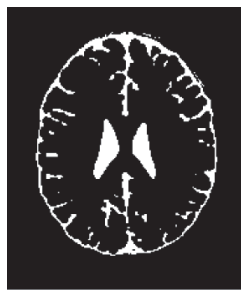

(s)

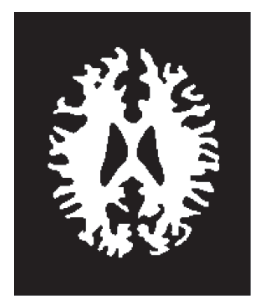

(d)

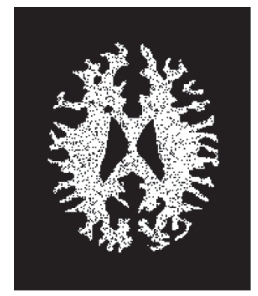

(j)

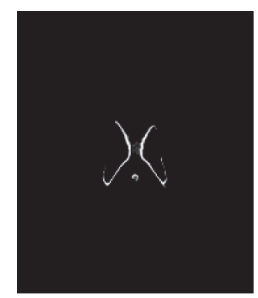

(p)

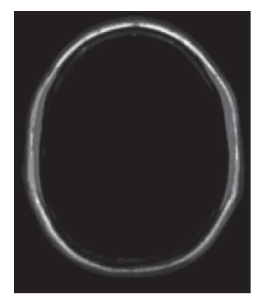

(t)

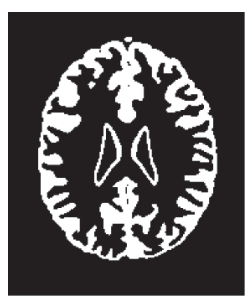

(e)

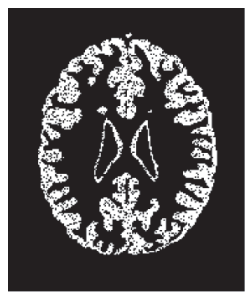

(k)

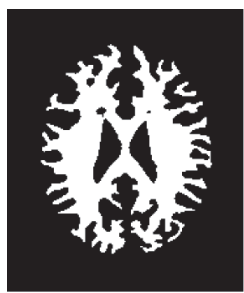

(q)

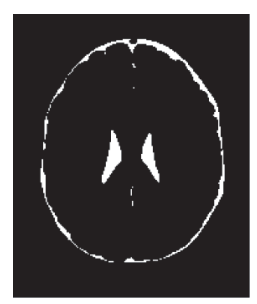

(f)

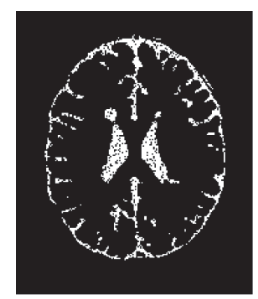

(1)

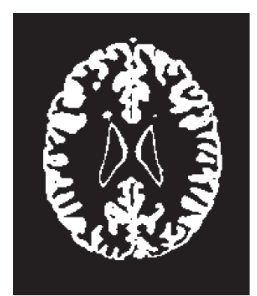

(r)

FIGURE 12: Segmentation comparisons when simulated MRI images have degraded by several processes. ((a) to (c)) Original images of T1weighted, T2-weighted, and $P D$-weighted images of the 98 th slice without skull regions. ((d) to (f)) By $3 \times 3$ averaging. ((g) to (i)) By Gaussian smoothing of $\mu=5$ and $\sigma=5$. ((j) to (l)) By adding 15\% uniform random noise. ((m) to (p)) Ground truth of segmentations: WM, GM, CSF, and glial matter. ((q) to (t)) Segmented result on original images without any noise corruption.

TABLE 4: Measured similarity of the proposed method, where $O I$ (original image) is degraded by $A 3(3 \times 3$ averaging filter), G5 (Gaussian smoothing of $\mu=5$ and $\sigma=5$ ), and UR (adding $15 \%$ uniform random noise).

\begin{tabular}{lcccc}
\hline \multirow{2}{*}{ Tissues } & \multicolumn{4}{c}{ Images } \\
& OI & A3 & G5 & UR \\
\hline$W M$ & 0.9214 & 0.8970 & 0.8473 & 0.7241 \\
$G M$ & 0.8472 & 0.8218 & 0.8147 & 0.6848 \\
CSF + glial matter & 0.8300 & 0.7534 & 0.7015 & 0.6273 \\
Average & 0.8662 & 0.8241 & 0.7878 & 0.6787 \\
\hline
\end{tabular}

the proposed method. The skull stripped images and the independent components extracted by the FastICA algorithm are, respectively, shown in Figures 14(d)-14(i). As indicated by the segmentation results presented in Figures 14(j)-14(m), the brain tissues were correctly extracted by the proposed ICKFCM algorithm.

\section{Conclusions}

The study proposes an ICKFCM method integrating skull stripping, ICA, and KFCM clustering for brain MRI image 


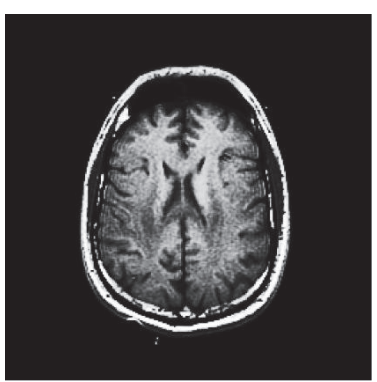

(a)

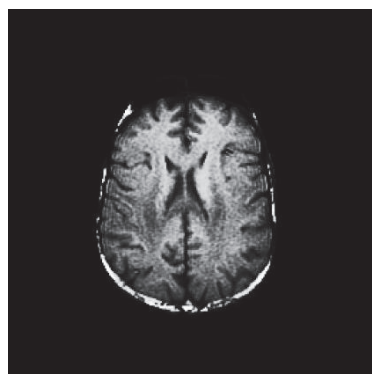

(d)

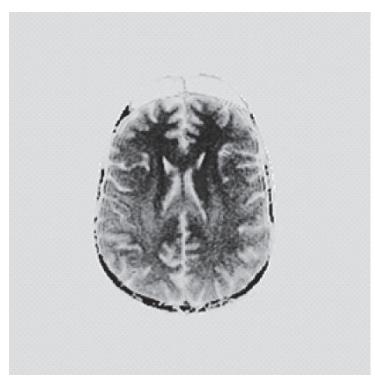

(g)

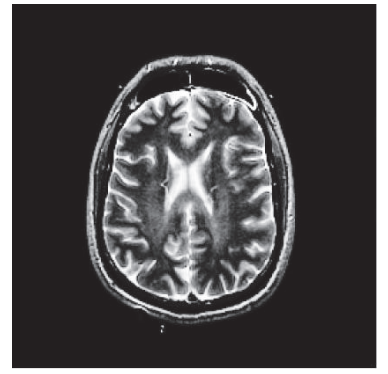

(b)

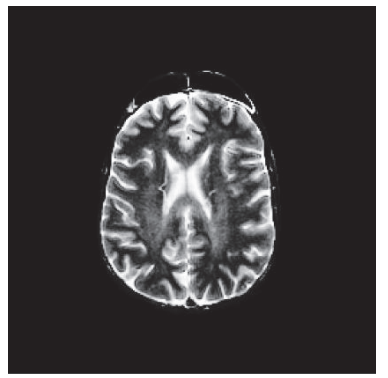

(e)

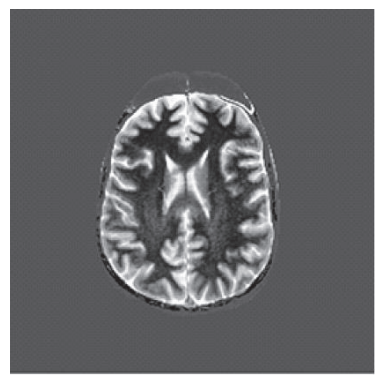

(h)

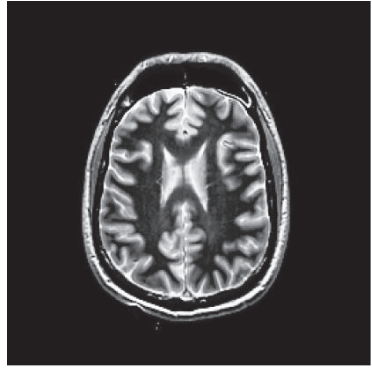

(c)

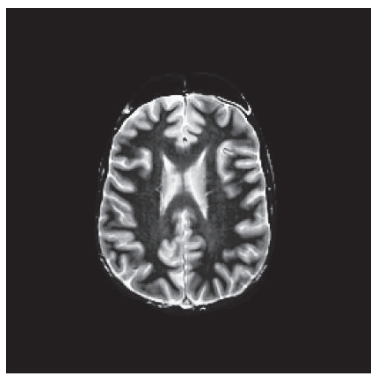

(f)

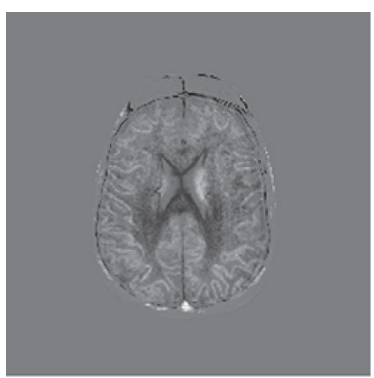

(i)

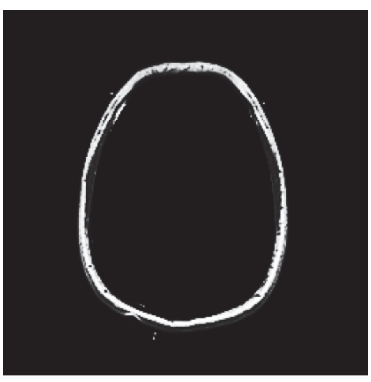

(j)

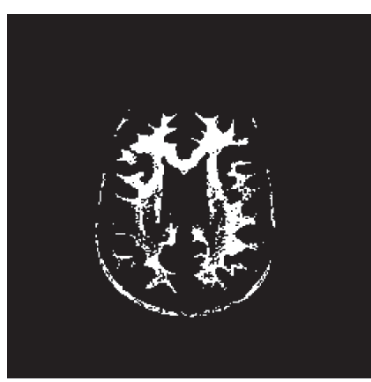

(k)

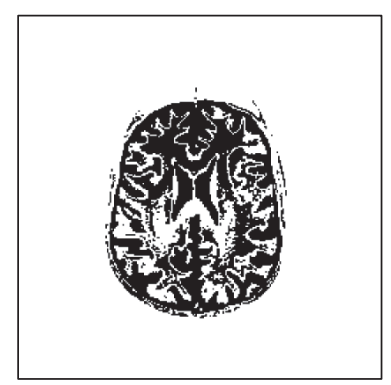

(l)

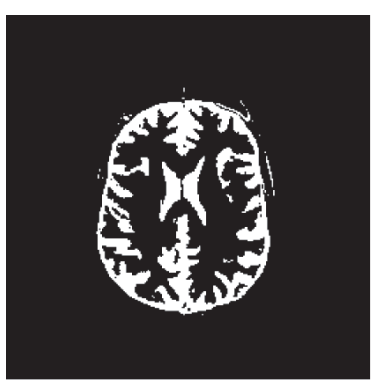

(m)

FIGURE 13: Segmentation on real medical images using the proposed method. ((a) to (c)) T1-weighted, T2-weighted, and PD-weighted MRI brain images. ((d) to (f)) Skull stripped images. ((g) to (i)) Three independent components extracted by FastICA algorithm. ((j) to (m)) Segmented results of skull region, $W M, G M$, and $C S F+$ glial matter.

segmentation. The skull region of the original images is firstly removed by a skull stripping algorithm. Since $M R I$ signals can be considered as a combination of the signals from each brain matter, with the FastICA algorithm, MRI images can be transformed into contrast-enhanced images (the independent components extracted by the FastICA algorithm). $K F C M$ is then used to segment the major brain tissues ( $W M$, $G M$, and CSF plus glial matter).
In the experiments, we discuss the segmentation performance of six methods ( $k$-means, FCM, KFCM, ICFCM, KWFLICM, and ICKFCM) for the simulated MRI images in noiseless case, noise case, and real medical images. To quantitatively compare the six segmentation methods, we further evaluate segmentation accuracy using JSI similarity measure. The results of both qualitative and quantitative comparison indicate that, compared to other segmentation methods, the 


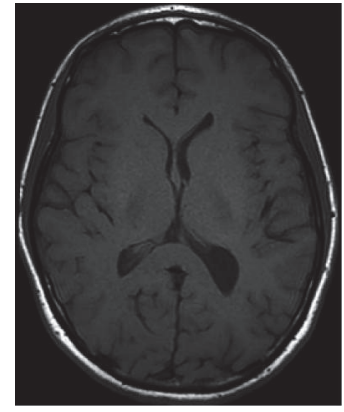

(a)

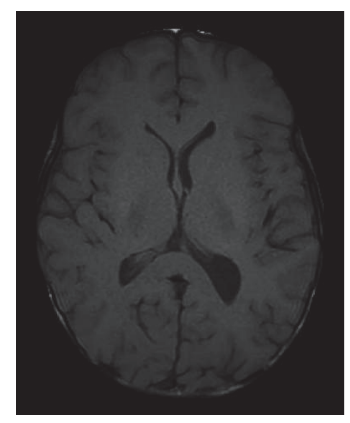

(d)

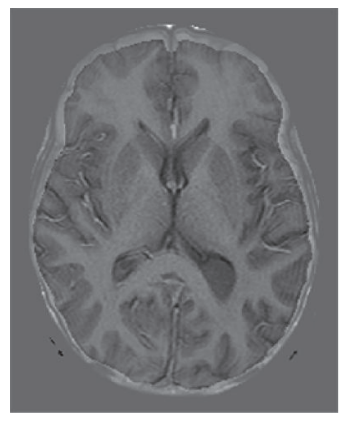

(g)

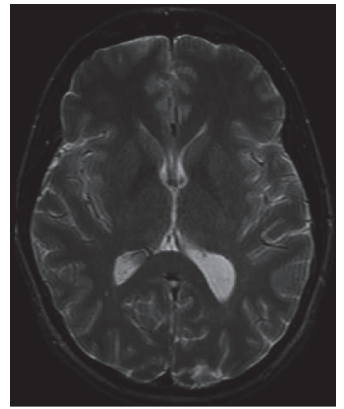

(b)

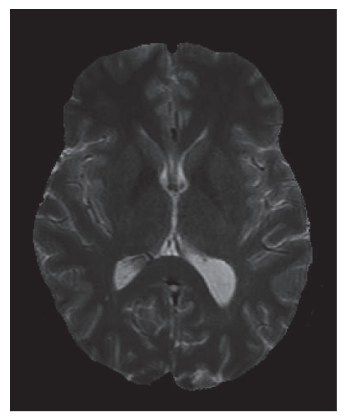

(e)

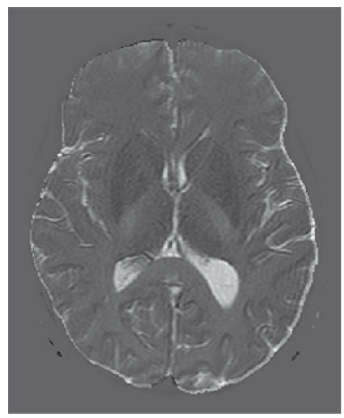

(h)

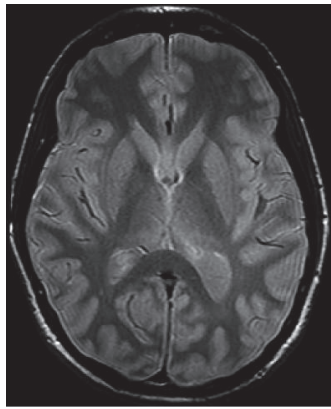

(c)

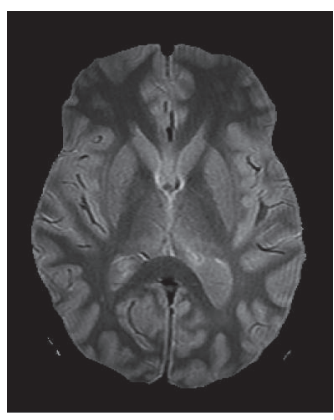

(f)

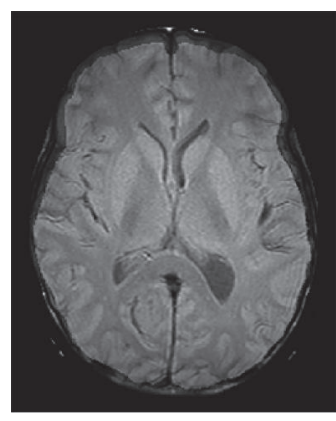

(i)

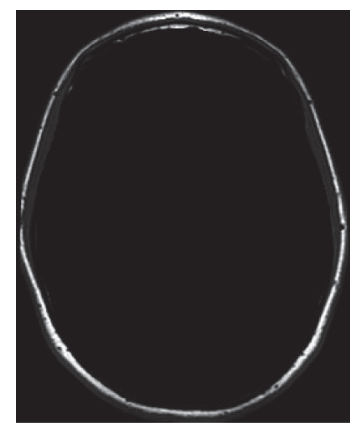

(j)

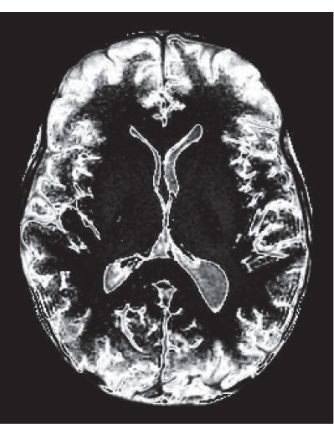

(k)

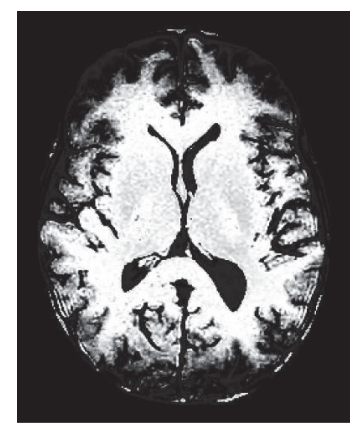

(1)

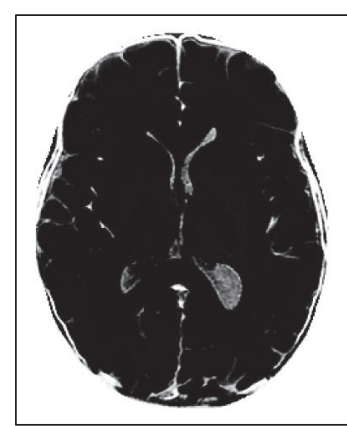

(m)

FIGURE 14: Segmentation on another real medical image using the proposed method. ((a) to (c)) T1-weighted, $T 2$-weighted, and $P D$-weighted MRI brain images. ((d) to (f)) Skull stripped images. ((g) to (i)) Three independent components extracted by FastICA algorithm. ((j) to (m)) Segmented results of skull region, $W M, G M$, and $C S F+$ glial matter. 
proposed ICKFCM method can extract the complicated shape of brain tissues and is robust against various types of noise.

\section{Competing Interests}

The author declares that there are no competing interests regarding the publication of this paper.

\section{References}

[1] P. R. Oliveira and R. A. F. Romero, "Improvements on ICA mixture models for image pre-processing and segmentation," Neurocomputing, vol. 71, no. 10-12, pp. 2180-2193, 2008.

[2] R. Jenssen and T. Eltoft, "Independent component analysis for texture segmentation," Pattern Recognition, vol. 36, no. 10, pp. 2301-2315, 2003.

[3] D. Gupta, R. S. Anand, and B. Tyagi, "A hybrid segmentation method based on Gaussian kernel fuzzy clustering and region based active contour model for ultrasound medical images," Biomedical Signal Processing and Control, vol. 16, pp. 98-112, 2015.

[4] Y.-T. Chen, "A level set method based on the Bayesian risk for medical image segmentation," Pattern Recognition, vol. 43, no. 11, pp. 3699-3711, 2010.

[5] Q. Zheng and E. Q. Dong, "Narrow band active contour model for local segmentation of medical and texture images," Acta Automatica Sinica, vol. 39, no. 1, pp. 21-30, 2013.

[6] G. Xiao, M. Brady, J. A. Noble, and Y. Zhang, "Segmentation of ultrasound B-mode images with intensity inhomogeneity correction," IEEE Transactions on Medical Imaging, vol. 21, no. 1, pp. 48-57, 2002.

[7] H.-M. Wu and H. H.-S. Lu, "Iterative sliced inverse regression for segmentation of ultrasound and MR images," Pattern Recognition, vol. 40, no. 12, pp. 3492-3502, 2007.

[8] W. M. Wells III, W. E. L. Grimson, R. Kikinis, and F. A. Jolesz, "Adaptive segmentation of MRI data," IEEE Transactions on Medical Imaging, vol. 15, no. 4, pp. 429-442, 1996.

[9] E. Sorouchyari, "Blind separation of sources, part III: stability analysis," Signal Processing, vol. 24, no. 1, pp. 21-29, 1991.

[10] T.-W. Lee, M. S. Lewicki, and T. J. Sejnowski, "ICA mixture models for unsupervised classification of non-Gaussian classes and automatic context switching in blind signal separation," IEEE Transactions on Pattern Analysis and Machine Intelligence, vol. 22, no. 10, pp. 1078-1089, 2000.

[11] A. J. Bell and T. J. Sejnowski, "An information-maximization approach to blind separation and blind deconvolution," Neural Computation, vol. 7, no. 6, pp. 1129-1159, 1995.

[12] A. Hyvärinen, J. Karhunen, and E. Oja, Independent Component Analysis, A Wiley-Interscience Publication, John Wiley \&Sons Inc, New York, NY, USA, 2001.

[13] T. Tateyama, Z. Nakao, and Y.-W. Chen, "Classification of brain matters in MRI by Kernel independent component analysis," in Proceedings of the 4th International Conference on Intelligent Information Hiding and Multimedia Signal Processing (IIH-MSP '08), pp. 713-716, IEEE, Harbin, China, August 2008.

[14] S. Wu, A. W. C. Liew, H. Yan, and M. Yang, "Cluster analysis of gene expression data based on self-splitting and merging competitive learning," IEEE Transactions on Information Technology in Biomedicine, vol. 8, no. 1, pp. 5-15, 2004.
[15] F. Masulli and A. Schenone, "A fuzzy clustering based segmentation system as support to diagnosis in medical imaging," Artificial Intelligence in Medicine, vol. 16, no. 2, pp. 129-147, 1999.

[16] G. Beni and X. Liu, "A least biased fuzzy clustering method," IEEE Transactions on Pattern Analysis and Machine Intelligence, vol. 16, no. 9, pp. 954-960, 1994.

[17] R. Krishnapuram and J. M. Keller, "Possibilistic approach to clustering," IEEE Transactions on Fuzzy Systems, vol. 1, no. 2, pp. 98-110, 1993.

[18] R. Krishnapuram and J. M. Keller, "The possibilistic C-means algorithm: insights and recommendations," IEEE Transactions on Fuzzy Systems, vol. 4, no. 3, pp. 385-393, 1996.

[19] D. Q. Zhang and S. C. Chen, "A novel kernelized fuzzy C-means algorithm with application in medical image segmentation," Artificial Intelligence in Medicine, vol. 32, no. 1, pp. 37-50, 2004.

[20] E. A. Zanaty, S. Aljahdali, and N. Debnath, "A kernelized fuzzy C-means algorithm for automatic magnetic resonance image segmentation," Journal of Computational Methods in Sciences and Engineering, vol. 9, no. 1, pp. 123-136, 2009.

[21] S. R. Kannan, S. Ramathilagam, R. Devi, and A. Sathya, "Robust kernel FCM in segmentation of breast medical images," Expert Systems with Applications, vol. 38, no. 4, pp. 4382-4389, 2011.

[22] M.-S. Yang and H.-S. Tsai, "A Gaussian kernel-based fuzzy cmeans algorithm with a spatial bias correction," Pattern Recognition Letters, vol. 29, no. 12, pp. 1713-1725, 2008.

[23] C. Li, C.-Y. Kao, J. C. Gore, and Z. Ding, "Implicit active contours driven by local binary fitting energy," in Proceedings of the IEEE Conference on Computer Vision and Pattern Recognition (CVPR '07), pp. 1-7, IEEE, Minneapolis, Minn, USA, June 2007.

[24] M. Gong, Y. Liang, J. Shi, W. Ma, and J. Ma, "Fuzzy C-means clustering with local information and kernel metric for image segmentation," IEEE Transactions on Image Processing, vol. 22, no. 2, pp. 573-584, 2013.

[25] E. A. Zanaty, "Determining the number of clusters for kernelized fuzzy C-means algorithms for automatic medical image segmentation," Egyptian Informatics Journal, vol. 13, no. 1, pp. 39-58, 2012.

[26] J. Kang, L. Min, Q. Luan, X. Li, and J. Liu, "Novel modified fuzzy C-means algorithm with applications," Digital Signal Processing, vol. 19, no. 2, pp. 309-319, 2009.

[27] D.-W. Kim, K. Lee, D. Lee, and K. H. Lee, "A kernel-based subtractive clustering method," Pattern Recognition Letters, vol. 26, no. 7, pp. 879-891, 2005.

[28] A. Hyvärinen and E. Oja, "A fast fixed-point algorithm for independent component analysis," Neural Computation, vol. 9, no. 7, pp. 1483-1492, 1997.

[29] E. A. Zanaty and A. Afifi, "Support vector machines (SVMs) with universal kernels," Applied Artificial Intelligence, vol. 25, no. 7, pp. 575-589, 2011.

[30] N. H. Sweilam, A. A. Tharwat, and N. K. Abdel Moniem, "Support vector machine for diagnosis cancer disease: a comparative study," Egyptian Informatics Journal, vol. 11, no. 2, pp. 81-92, 2010.

[31] BrainWeb. Simulated Brain Database, http://brainweb.bic.mni .mcgill.ca/.

[32] National Library of Medicine, 2016, http://www.nlm.nih.gov/.

[33] Department of Medical Imaging and Radiological Technology, Yuanpei University of Medical Technology, 2016, http:// rad.ypu.edu.tw/.

[34] R. Cárdenes, R. de Luis-García, and M. Bach-Cuadra, "A multidimensional segmentation evaluation for medical image data," Computer Methods and Programs in Biomedicine, vol. 96, no. 2, pp. 108-124, 2009. 


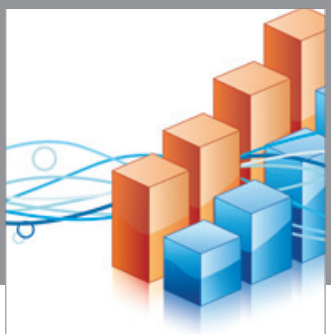

Advances in

Operations Research

vatem alat4

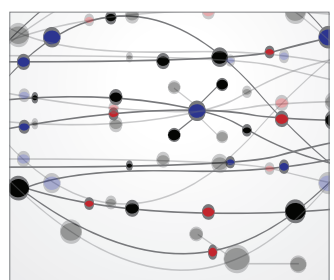

\section{The Scientific} World Journal
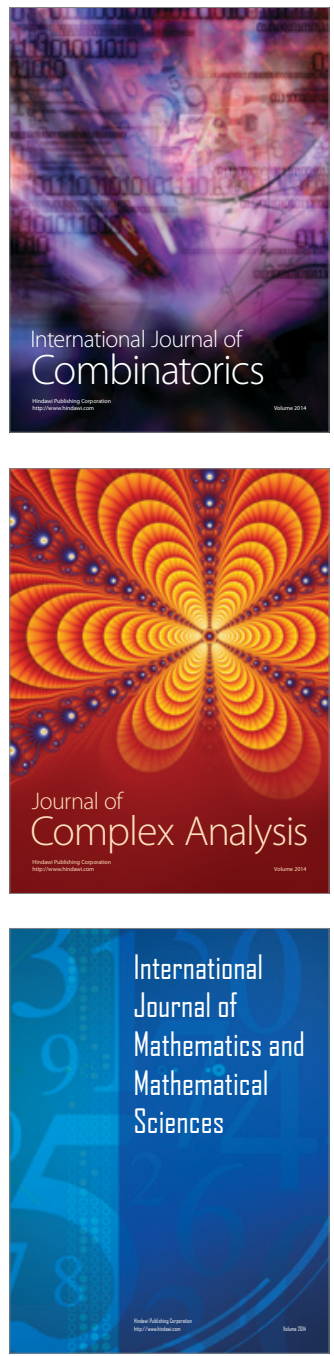
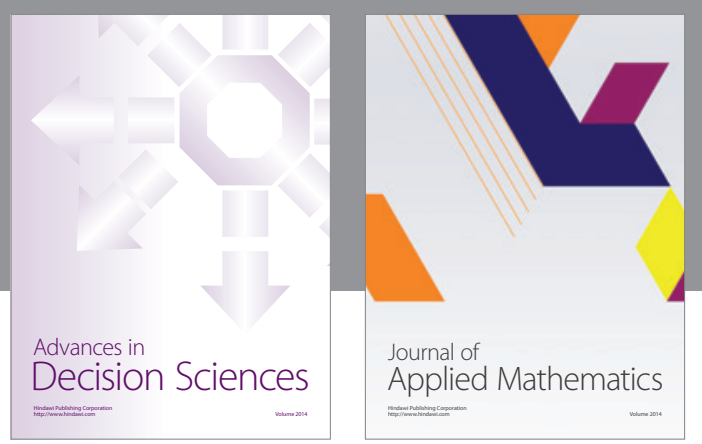

Algebra

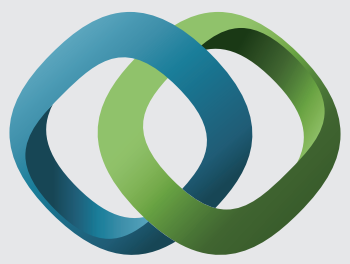

\section{Hindawi}

Submit your manuscripts at

https://www.hindawi.com
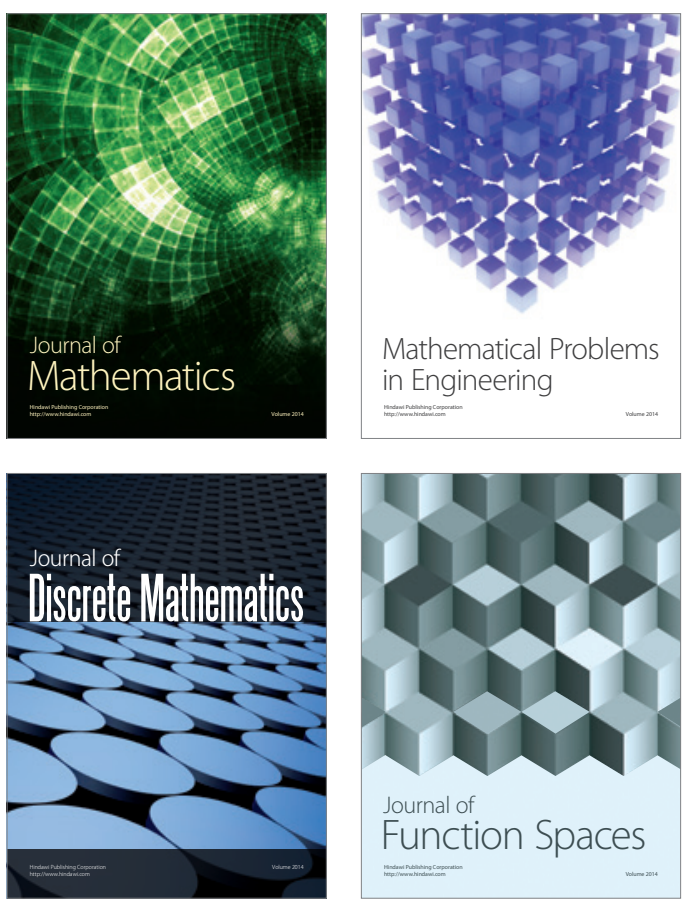

Mathematical Problems in Engineering
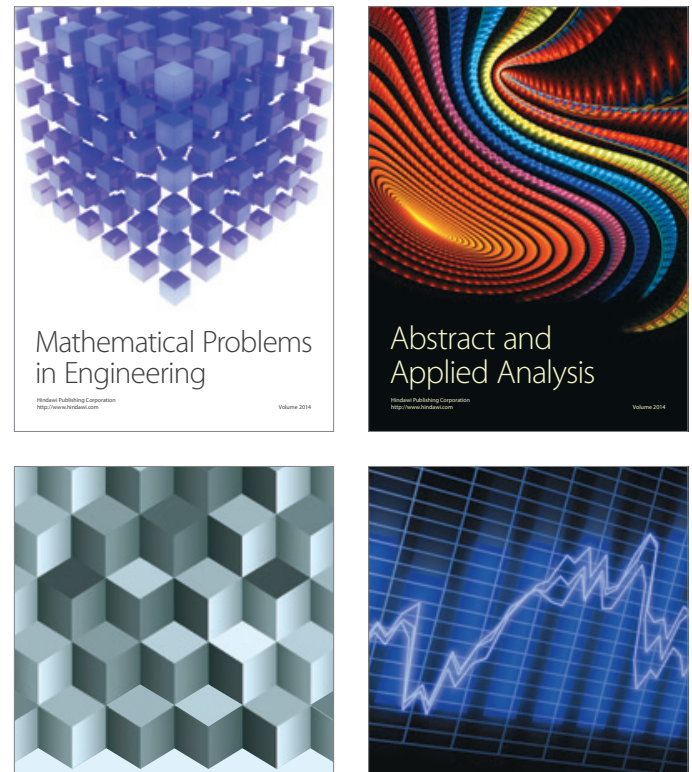

Journal of

Function Spaces

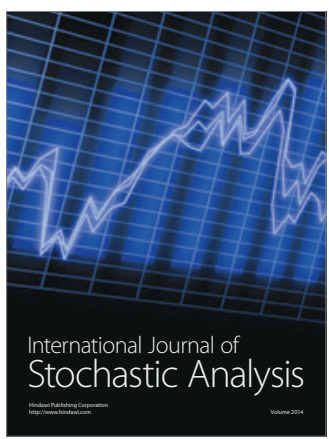

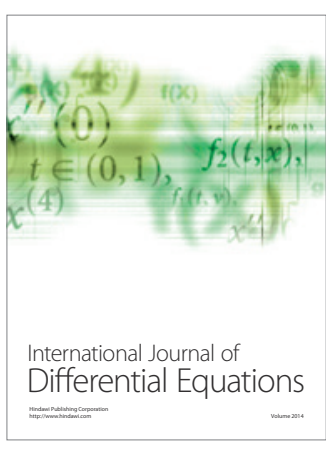
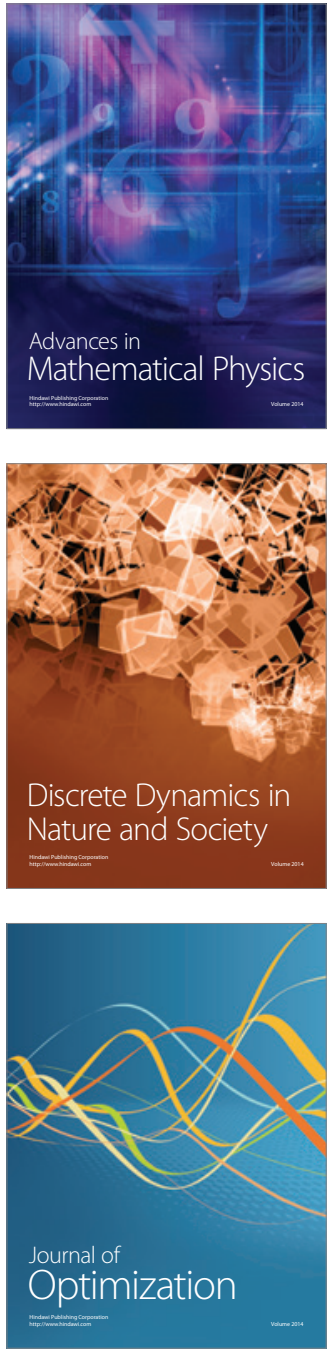